\title{
Benchmarking ideal sample thickness in cryo-EM using
} MicroED

\author{
Michael W. Martynowycz ${ }^{1,2}$, Max T.B. Clabbers ${ }^{2}$, Johan Unge ${ }^{2}$, Johan Hattne ${ }^{1,2}$ and \\ Tamir Gonen ${ }^{1,2,3 *}$
}

\begin{abstract}
${ }^{1}$ Howard Hughes Medical Institute, University of California Los Angeles, Los Angeles CA 90095

2 Department of Biological Chemistry, University of California Los Angeles, Los Angeles CA 90095
\end{abstract}

${ }^{3}$ Department of Physiology, University of California Los Angeles, Los Angeles CA 90095 To whom correspondence should be sent: tgonen@g.ucla.edu

\section{Abstract}

The relationship between sample thickness and quality of data obtained by microcrystal electron diffraction (MicroED) is investigated. Several EM grids containing proteinase $\mathrm{K}$ microcrystals of similar sizes from the same crystallization batch were prepared. Each grid was transferred into a focused ion-beam scanning electron microscope (FIB/SEM) where the crystals were then systematically thinned into lamellae between $95 \mathrm{~nm}$ and $1650 \mathrm{~nm}$ thick. MicroED data were collected at either 120, 200, or $300 \mathrm{kV}$ accelerating voltages. Lamellae thicknesses were converted to multiples of the calculated inelastic mean free path (MFP) of electrons at each accelerating voltage to allow the results to be compared on a common scale. The quality of the data and subsequently determined structures were assessed using standard crystallographic measures. Structures were reliably determined from crystalline lamellae only up to twice the inelastic mean free path. Lower resolution diffraction was observed at three times the mean free path for all three accelerating voltages but the quality was insufficient to yield structures. No diffraction data 
were observed from lamellae thicker than four times the calculated inelastic mean free path. The quality of the determined structures and crystallographic statistics were similar for all lamellae up to $2 x$ the inelastic mean free path in thickness, but quickly deteriorated at greater thicknesses. This study provides a benchmark with respect to the ideal limit for biological specimen thickness with implications for all cryo-EM methods.

\section{Significance}

A systematic investigation of the effects of thickness on electron scattering from protein crystals was previously not feasible, because there was no accurate method to control sample thickness. Here, the recently developed methods for preparing protein crystals into lamellae of precise thickness by ion-beam milling are used to investigate the effects of increasing sample thickness on MicroED data quality. These experiments were conducted using the three most common accelerating voltages in cryo-EM. Data across these accelerating voltages and thicknesses were compared on a common scale using their calculated inelastic mean free path lengths. It is found that structures may accurately be determined from crystals up to twice the inelastic mean free path length in thickness, regardless of the acceleration voltage.

\section{Main}

High energy electrons interact strongly with matter (1-3). This property has enabled a revolution in structural biology by electron cryo-microscopy (cryo-EM) techniques (4). However, this strong interaction also implies a higher probability of an electron scattering multiple times and/or losing energy within the specimen (5). The probability of scattering relates to a physical property known as the mean free path. This is the average distance travelled through a sample by a moving particle before an interaction takes place. The inelastic mean free path (MFP) refers to the typical distance a high-energy electron travels through a specimen before losing energy, or inelastically scattering. In cryo-EM, the MFP is often used to compare samples of different thicknesses across different accelerating voltages $(6,7)$. The MFP in cryo-EM may be roughly calculated for a given sample, and 
57 has been investigated experimentally in vitreous ice, since this is the most probable 58 environment in these experiments, though similar values have recently been demonstrated in liquid water $(7,8)$. The calculated MFP for a typical protein crystal at accelerating voltages of 120,200 or $300 \mathrm{kV}$ would correspond to roughly to 214,272 and $317 \mathrm{~nm}$, respectively (Materials and Methods).

Early cryo-EM investigations of electron diffraction from frozen-hydrated protein samples reported measurable differences between the intensities of Friedel mates from twodimensional crystals of bacteriorhodopsin (bR) (9). These differences were suggested to arise from dynamically scattered electrons. Dynamically scattered electrons could introduce significant errors, breaking the relationship between the recorded intensity and the underlying structure factor amplitude. Computational simulations suggested that twodimensional crystals of bR thicker than $20 \mathrm{~nm}$ at $100 \mathrm{kV}$, and three-dimensional crystals of lysozyme thicker than $100 \mathrm{~nm}$ at $200 \mathrm{kV}$ would result in highly inaccurate intensities due to dynamical scattering $(10,11)$. Those results are at odds with earlier reports that diffraction intensities from three-dimensional catalase crystals at $200 \mathrm{kV}$ scatter kinematically at thicknesses up to at least $150 \mathrm{~nm}$ (12). Indeed, investigations reported structures of catalase from crystals of variable crystal thicknesses without the need of any dynamical corrections $(13,14)$. Many macromolecular structures have since been reported from crystals that are thicker than $100 \mathrm{~nm}$ using the cryo-EM method microcrystal electron diffraction (MicroED) (14-20).

Until recently, a systematic investigation of how crystal thickness effects data quality was not feasible. Now, FIB milling allows the thickness of a protein crystal to be precisely controlled (21-26). This process is similar to milling cellular and tissue specimens to prepare them for subsequent cryo-tomography investigations $(21,23,27,28)$. Zhou et al. recently used this technique to mill several crystals to different thicknesses and compared single diffraction pattern from each at $200 \mathrm{kV}$ (24). However, they did not systematically correlate the effect of crystal thickness on the ability to determine structures. Only a systematic investigation of integrated intensities and the quality of the determined protein 
structures from lamellae of variable thicknesses would shed light on the role of sample thickness in cryo-EM and possible errors due to dynamical scattering.

Here, we investigate the impact of sample thickness on the ability to determine structures and the quality of data obtained using MicroED. Microcrystals of proteinase K were milled into lamellae between 95 and $1650 \mathrm{~nm}$ thick. MicroED data were collected from each lamella at one of the three most common acceleration voltages (120, 200, and $300 \mathrm{kV})$ (Figure 1). The data were put on a common scale by relating their thicknesses to the inelastic mean free path at their respective acceleration voltage. These thicknesses roughly correspond to between $0.5 \times$ and $5 \times$ MFP. We found that MicroED data from crystals as thick as twice the MFP are sufficiently accurate to determine high-resolution protein structures irrespective of the acceleration voltage. Surprisingly, no large difference in data or structure quality was observed from lamellae below $2 \times$ MFP. Diffraction was still observed at up to $3 \times$ MFP, but the data was not suitable for processing. No diffraction spots were observed for thicknesses beyond 4× MFP. These trends were true for all three acceleration voltages. This study provides initial measurements of crystals of definitive thicknesses at varying accelerating voltages and provides limits on biological specimen thickness with implications for all cryo-EM investigations.

\section{Results}

Preparing grids with protein crystals. Proteinase K crystals were grown in batch as described $(29,30)$. This condition results in protein microcrystals that measure between 10 and $30 \mu \mathrm{m}$ across their middle. TEM grids were prepared by back blotting as described (30). The samples were then loaded into a focused ion-beam scanning electron microscope (FIB/SEM).

111 Vitrified grids were coated with sputtered platinum to protect the crystals from the 112 damaging electron and ion-beams during investigation (29) (Materials and Methods). 113 Each grid was searched using the scanning electron beam for crystals that satisfied the 114 following requirements: each crystal was of relatively similar size, was at least $5 \mu \mathrm{m}$ away 
115 from the nearest grid bar, and at least 3 grid squares away from the edge of the grid. In

116 this way, 5 appropriate crystals were identified on a first batch of grids, 9 on the second, 117 and 7 on the third. Each crystal was inspected in both the SEM and FIB and aligned to 118 eucentric height. Lamellae were milled by rastering the gallium beam across the 119 microcrystals (Materials and Methods). The ion-beam current was lowered as the desired thickness was approached as described $(27,29)$. The thickness of each crystalline

121 lamella was measured by taking an image in the ion-beam just prior to unloading each 122 grid. Lamellae thicknesses spanned from approximately 95 to $1650 \mathrm{~nm}$ (Figure 1, Figure 2) (Materials and Methods).

MicroED experiments on crystalline lamellae. Each grid was carefully rotated by $90^{\circ}$ when loading into the TEM, such that the rotation axis in the TEM was perpendicular to the milling direction in the FIB/SEM. Samples were investigated at an accelerating voltage of either 120,200 , or $300 \mathrm{kV}$. Lamellae on each grid were identified by low-magnification montaging, and the eucentric height was adjusted individually for each site. A selected area aperture was used to isolate the diffraction from a circular area approximately $3 \mu \mathrm{m}$ in diameter from the center of each lamella. In this way, no diffraction or signal from anything other than the flat, thickness-controlled lamellae would be recorded during data collection. Continuous rotation MicroED data were collected from the real space wedge between $30^{\circ}$ and $-30^{\circ}$ from each lamella (Figure 3 ).

An estimate of resolution for a complete dataset can be obtained by visually inspecting the projection of maximum intensities through an entire continuous rotation dataset. We calculated these projections for all the lamellae (Supplementary Figures 1 - 36). These estimates corroborate the general trends seen in the integration statistics: high quality signal clearly persists to $2 \times$ MFP, greatly diminished by $3 \times$ MFP, and completely lost at approximately $4 \times$ the MFP and beyond. Measurements at 5× MFP were also taken and found to be similarly void of diffraction suggesting that at these thicknesses electrons are fully absorbed by the samples (SI Figures 24, 36).

142 Data from each lamella were converted to crystallographic format (Figure 3, 143 Supplementary Figures 1 - 36). These datasets were then indexed, integrated, and scaled 144 individually as described (Materials and Methods) (31). A resolution cutoff was applied to 
145

146

147

148

149

150

151

152

153

154

155

156

157

158

159

160

161

162

163

164

165

166

167

168

169

170

171

172

173

174

each dataset where the $\mathrm{CC}_{1 / 2}$ value fell to $\sim 33 \%$ (Table 1)(32). Integration statistics are reported for each lamella in Table 1. Collectively, strong and sharp reflections were observed from crystal lamellae that were up to $2 \times$ MFP thick at the three acceleration voltages. Little or poor diffraction was observed at $3 \times$ MFP while above $4 \times$ MFP no diffraction could be observed (Figure 3). Lamellae that yielded usable data were individually integrated and their respective models refined (Table 1, Supplementary Tables 1). In all cases, the calculated maps and composite omit maps were of high quality (Figure 4, Supplementary Figure 37).

\section{Discussion}

The relationship between crystal thickness, acceleration voltage, and the quality of MicroED data and the subsequently determined structures was systematically investigated. The samples used here were of the same protein, grown in the same batch, from crystals of originally similar size, and machined into lamellae using the same protocol (Materials and Methods). MicroED data were collected using established procedures (17, $23,33)$. The data were collected at the three most common acceleration voltages used in cryo-EM - 120, 200, and 300kV. No increasing, systematic errors prevented structure determination from crystals up to twice the MFP. Even thinner crystals did not appear to have any significant advantage (Figure 5, Table 1). Indeed, the crystallographic statistics, metrics of the determined structures, and resulting maps from lamellae up to $2 \times$ MFP all appear relatively similar (Table 1 and Figure 5). However, beyond $2 \times$ MFP the overall resolution and quality metrics rapidly fall off (Figure 5). No structures could be determined at $3 \times$ MFP for all acceleration voltages, although sporadic diffraction spots at low resolution were visible on some images. No diffraction was observed for samples thicker than $3 \times$ MFP, suggesting that most electrons are absorbed by the sample. These observations are in agreement with the measurements of single diffraction patterns from Zhou et al. (24), where the best data were observed at intermediate thicknesses rather than at an extremum.

The data suggests that the largest detriment to determining structures by MicroED from thicker samples appears to be absorption. Reliable high quality structures can be 
175 determined from up to $2 \times$ MFP regardless of acceleration voltage. Beyond that point, the 176 resolution deteriorated and structures became difficult to determine. Absorption effects 177 are compounded by the fact that thicker crystals produce more diffuse scattering, and 178 increased inelastic scattering results in higher background noise. Taken together, it is 179 clear that the elastic, Bragg peaks are diminished by absorption in thick samples, and 180 that the higher resolution reflections are quickly lost to additional noise from the increased background. It is possible that an energy filter could help mitigate some of these issues, and we expect that the usable thickness could slightly increase with this addition. Initial results already demonstrated that an energy filter leads to an increase in the signal to noise ratio and the attainable resolution (34-38).

Computer simulations are at odds with MicroED experimental results. All simulations are inherently limited by the validity of their assumptions, as correctly discussed in Subramanian et al. (10). We suggest that at least five assumptions are inadequate in current electron diffraction simulations. Namely most assume that: 1. macromolecular crystals are perfect; 2 . diffraction is only collected from a major zone axis (always true for 2D crystals, but rarely for 3D crystals); 3. contribution from disordered solvent is negligible 4. data are collected from stationary crystals (MicroED uses continuous rotation) and 5. inelastic scattering, or absorption is not significant. Though more recent simulations have made progress in accounting for some of these discrepancies (39), future simulations would benefit from a more accurate modelling of the experimental setup.

We demonstrated that high quality structures can be obtained from samples that are up to $2 \times$ the MFP regardless of the acceleration voltage. This corresponds to a real space thicknesses of approximately 430,540, and $640 \mathrm{~nm}$ for the accelerating voltages of 120, 200 , and $300 \mathrm{kV}$, respectively. We find that samples that were thicker did not yield usable data, and electrons were generally lost to absorption at thicknesses of $4 \times$ MFP or more. We expect that these limits could be somewhat relaxed with an energy filter, but the exact parameters will need to be investigated in future studies. Importantly, as FIB milling becomes a standard method for sample preparation for macromolecular MicroED studies, aiming for a thicknesses of less than $2 \times$ MFP will maximize the likelihood of producing the best data and highest quality structures. Regardless of the cryo-EM method employed, 
205 the current study provides a benchmark for the sample thickness in cryo-EM especially

206 for electron tomographic studies of FIB milled tissues and cells.

207

208

209

210

211

212

213

214

215

216

217

218

219

220

221

222

223

224

225

226

227

228

229

230

231

232

\section{Acknowledgements}

This study was supported by the National Institutes of Health P41GM136508. The Gonen laboratory is supported by funds from the Howard Hughes Medical Institute.

\section{Materials and Methods}

Materials. Proteinase K (E. Album) and ammonium sulfate were purchased from Sigma (Sigma-Aldrich, St. Louis, MO) and used without further purification. Stock solutions were made using Milli-Q water filtered three times through a $0.4 \mu \mathrm{m}$ porous membrane.

Crystallization. Proteinase K was crystallized in batch by dissolving $1 \mathrm{mg}$ of lyophilized protein powder with $200 \mu \mathrm{L}$ of $2 \mathrm{M}$ ammonium sulfate at room temperature. 10-30 $\mu \mathrm{m}$ crystals formed within 10 minutes.

Grid preparation. Quantifoil Cu 200 R 2/2 grids were glow discharged for $30 \mathrm{~s}$ immediately prior to use. Grids were vitrified using a Leica GP2 vitrification robot at room temperature. The sample chamber was set to $95 \%$ relative humidity and the filter paper equilibrated in the humid air for 15 min prior to grid preparation. The tube of crystals was gently shaken just before $3 \mu \mathrm{L}$ of protein crystal solution was removed and gently pipetted onto the carbon side of the grid in the vitrification chamber. The slurry was incubated on the grid for $30 \mathrm{~s}$. The grid was then gently blotted from the back for $20 \mathrm{~s}$, plunged into liquid ethane, and transferred to liquid nitrogen for storage.

Focused ion-beam and scanning electron microscopy. Vitrified grids were transferred into a cryogenically cooled Thermo-Fisher Aquilos dual beam FIB/SEM. The grids were coated in a thin layer of fine platinum followed by a thick $>100 \mathrm{~nm}$ layer of coarse platinum by sputter coating to protect the crystals from the damaging ion and electron beams (29). Whole grid atlases were recorded using the MAPS software (Thermo-Fisher), where individual crystals were identified and aligned to eucentric height. Twenty-one crystals 
233

234

235

236

237

238

239

240

241

242

243

244

245

246

247

248

249

250

251

252

253

254

255

256

257

258

259

260

261

262

263

over six grids were chosen for milling. Crystals were milled as described $(23,29,30,40$, 41). Briefly, the milling was conducted in steps of: rough milling, fine milling, and polishing. Each step was performed on each crystal sequentially prior to advancing to the next step to reduce the effects of amorphous ice buildup and contamination. Rough milling steps were conducted at $100 \mathrm{pA}$ and removed all but $5 \mu \mathrm{m}$ of crystalline material. Fine milling used an ion-beam current of $50 \mathrm{pA}$ and removed material up to $250 \mathrm{~nm}$ away from the desired thickness. Polishing was conducted at an ion-beam current of $10 \mathrm{pA}$ and was used until the approximate final thickness was achieved. A final image of the lamella was taken using the ion-beam at $1.5 \mathrm{pA}$ (Figure 2, Supplementary Figures $1-36$ ) to assess the final thickness using the measurement tool in the Aquilos user interface. All micrographs taken by the scanning electron beam were performed at an accelerating voltage of $5 \mathrm{kV}$ and a beam current of $1.6 \mathrm{pA}$. All ion-beam imaging and milling was conducted at an accelerating voltage of $30 \mathrm{kV}$.

MicroED data collection. Grids containing milled lamellae were transferred to either a Thermo-Fisher Titan Krios G3i or Talos Arctica transmission electron microscopes. The Krios was operated at an accelerating voltage of either 120 or $300 \mathrm{kV}$, whereas the Arctica operated at an accelerating voltage of $200 \mathrm{kV}$. Electrons at the accelerating voltages of 120,200 or $300 \mathrm{kV}$ have corresponding de Broglie wavelengths of $0.0335 \AA, 0.0251 \AA$, or $0.0197 \AA$, respectively. Both microscopes were equipped with a field emission gun and a Ceta-D 16M (4k×4k) CMOS detector. Lamellae were identified on each grid by taking a low-magnification montage, where they appeared as thin white stripes against an otherwise dark background. Crystalline lamellae within these strips appeared semitransparent and suspended over this gap. Lamellae were brought to eucentric height and initially evaluated by taking a single $1 \mathrm{~s}$ exposure in diffraction mode at $0^{\circ}$ stage tilt. MicroED data were collected as described (33). In short, the stage was continuously rotated at a rate of $0.25^{\circ} \mathrm{s}^{-1}$ while the crystal was illuminated in a parallel electron beam. Frames were read out from the detector every 1 or 2 seconds and saved as a stack in MRC format. Each dataset corresponded to 120 - 240 images corresponding to the realspace angular wedge between +30 to $-30^{\circ}$. The electron beam was approximately $10 \mu \mathrm{m}$ in diameter, where the corresponding exposure was calibrated to a rate of approximately $0.01 \mathrm{e}^{-} \AA^{-2} \mathrm{~s}^{-1}$. The total exposure to each lamellae was therefore approximately $2.4 \mathrm{e}^{-} \AA^{-}$ 
2642 . Signal from the center of each lamella was isolated by inserting a selected area aperture 265 of $100 \mu \mathrm{m}$ for the Arctica or $150 \mu \mathrm{m}$ for the Krios, corresponding to an area of approximately $3 \mu \mathrm{m}$ in diameter projected from the sample for either microscope.

267

268

269

270

271

272

273

274

275

276

277

278

279

280

281

282

283

284

285

286

287

MicroED data processing. MRC stacks were converted to individual frames in SMV format using the MicroED tools as described $(23,33)$. MicroED tools can be downloaded freely at (https://cryoem.ucla.edu/downloads). Reflections were indexed and integrated in XDS (31). Individual or groups of datasets were scaled in either AIMLESS (42) or XSCALE (43). Each dataset was used to determine the structure using molecular replacement in Phaser with the search model PDB 6CL7 $(44,45)$. Models were refined in phenix.refine (46) using electron scattering factors.

Calculations. The inelastic mean free path (MFP) was calculated using the original formulation by Malis et al. (6) and Egerton (47) et al. and subsequently used by Feja et al. (48) and Grimm et al. (8) given by:

and

$$
\Lambda=\frac{106 F\left(\frac{E_{0}}{E_{m}}\right)}{\ln \left(\frac{2 \beta E_{0}}{E_{m}}\right)}
$$

where

$$
F=\frac{\left(1+\frac{E_{0}}{1022}\right)}{\left(1+\frac{E_{0}}{511}\right)^{2}}
$$

$$
E_{m}=7.6 Z^{0.36}
$$

We similarly used the values of $\beta=10 \mathrm{mrad}, Z=8$, and $E_{0}$ for the acceleration voltage used, e.g. 120, 200, or $300 \mathrm{kV}$ as the typical values employed in those investigations.

An optical refractive term was applied as suggested in Grimm et al. (8), and a value of $n$ $=1.48$ was chosen based on the determined value for lysozyme layers (49). This translates to inelastic mean free path values of 214, 272, and $317 \mathrm{~nm}$ for 120,200 , and $300 \mathrm{kV}$ accelerating voltages. These values are in good agreement with those previously 
288

289

290

291

292

293

294

295

296

297

298

299

300

301

302

303

304

305

306

307

308

309

310

measured experimentally $(6-8,47,48,50)$ and have a direct relationship to the deposited dose (51).

The mean-free path is closely related to the collision stopping power, as calculated using e.g. ESTAR (52). For a typical protein sample with density $1.17 \mathrm{~g} \mathrm{~cm}^{-3}(53)$, the tabulated stopping power implies that a $120 \mathrm{kV}$ electron loses $4.15 \mathrm{MeV}$ per $\mathrm{cm}$ of traversed sample (3.22 MeV and 2.72 MeV for $200 \mathrm{kV}$ and $300 \mathrm{kV}$ electrons, respectively). The deposited energy per unit length calculated by this method was previously used to derive estimates of the dose from a given exposure $(45,51)$ and its inverse is correlated to the mean-free path length used here with an asymptotic standard error of $<2 \%$.

The curves presented in Figure $5(B)$ and $(C)$ are reverse sigmoid functions of the form:

$$
f(x)=a * \frac{1}{1+e^{b(x-c)}}
$$

The functions were fit using simple least squares.

\section{Table and figure legends}

Figure 1. Preparation of protein microcrystals into lamellae of specified thicknesses. Schematic cartoon showing the general process of systematically investigating data quality for variably thick samples. Crystals are identified on EM grids (top), milled to specified thicknesses (middle), and MicroED datasets are collected from each crystal at either 120,200 , or $300 \mathrm{kV}$ accelerating voltages.

Figure 2. Preparing protein lamellae of variable thicknesses. Images taken by the gallium ion-beam of selected crystalline lamellae after milling. Images are sorted into rows and columns by the accelerating voltages used for data collection, and the calculated multiple of the inelastic mean free path for protein for that condition. Approximate location and size of lamellae are indicated by blue arrows and blue lines.

Figure 3. MicroED data from lamellae of different thicknesses and accelerating voltages. Single frames from MicroED datasets corresponding to $0^{\circ}$ tilt. Frames are sorted into columns by the accelerating voltage and rows for the approximate thickness 
expressed as a multiple of the calculated inelastic mean free path at that accelerating voltage.

Figure 4. MicroED structures determined from lamellae of specified thicknesses. (Top) Final structure solution of Proteinase $\mathrm{K}$ in rainbow with a loop corresponding to residues 126-132 shown as gray spheres. (Bottom) $2 m F_{o}-F_{c}$ maps from lamellae of different thicknesses and resolution cutoffs for the selected loop from above. All maps contoured at $1.5 \sigma$ level with a $2 \AA$ carve.

Figure 5. Data quality metrics as a function of thickness. (A) Resolution cutoff, (B) mean signal to noise, and $(\mathrm{C})$ the half set correlation coefficient for all the measured crystals. Data points are color coded according to accelerating voltage as indicated. A simple reverse sigmoid function is fit to the data in $(B)$ and $(C)$ to demonstrate the sharp drop in quality after approximately $2 \times$ MFP. $\left({ }^{*}\right)$ Value approximated from diffraction images that could not be automatically processed. $\left({ }^{* *}\right)$ Best possible value given experimental cutoff due to beamstop. $\left(^{* * *}\right)$ Value presumed from inability to integrate datasets

\section{Table 1. MicroED data from lamellae of different thicknesses}

\section{References}

1. J. M. Cowley, A. F. Moodie, The scattering of electrons by atoms and crystals. I. A new theoretical approach. Acta Cryst 10, 609-619 (1957).

2. R. Henderson, The potential and limitations of neutrons, electrons and X-rays for atomic resolution microscopy of unstained biological molecules. Quarterly reviews of biophysics 28, 171-193 (1995).

3. B. K. Vainshtein, E. Feigl, J. A. Spink, Structure Analysis by Electron Diffraction. (Elsevier Science, 2014) (May 11, 2021).

4. W. Kuhlbrandt, The Resolution Revolution. Science 343, 1443-1444 (2014).

5. K. Fujiwara, Application of Higher Order Born Approximation to Multiple Elastic Scattering of Electrons by Crystals. J. Phys. Soc. Jpn. 14, 1513-1524 (1959).

6. T. Malis, S. C. Cheng, R. F. Egerton, EELS log-ratio technique for specimen-thickness measurement in the TEM. J. Elec. Microsc. Tech. 8, 193-200 (1988). 
7. M. N. Yesibolati, et al., Electron inelastic mean free path in water. Nanoscale 12, 20649-20657 (2020).

8. R. Grimm, D. Typke, M. Bärmann, W. Baumeister, Determination of the inelastic mean free path in ice by examination of tilted vesicles and automated most probable loss imaging. Ultramicroscopy 63, 169-179 (1996).

9. R. M. Glaeser, T. A. Ceska, High-voltage electron diffraction from bacteriorhodopsin (purple membrane) is measurably dynamical. Acta Crystallogr A Found Crystallogr 45, 620-628 (1989).

10. G. Subramanian, S. Basu, H. Liu, J.-M. Zuo, J. C. H. Spence, Solving protein nanocrystals by cryo-EM diffraction: Multiple scattering artifacts. Ultramicroscopy 148, 87-93 (2015).

11. R. M. Glaeser, K. H. Downing, High-resolution electron crystallography of protein molecules. Ultramicroscopy 52, 478-486 (1993).

12. D. L. Dorset, D. F. Parsons, Electron diffraction from single, fully-hydrated, ox-liver catalase microcrystals. Acta Cryst A 31, 210-215 (1975).

13. B. L. Nannenga, D. Shi, J. Hattne, F. E. Reyes, T. Gonen, Structure of catalase determined by MicroED. elife 3, e03600 (2014).

14. K. Yonekura, K. Kato, M. Ogasawara, M. Tomita, C. Toyoshima, Electron crystallography of ultrathin 3D protein crystals: atomic model with charges. Proceedings of the National Academy of Sciences 112, 3368-3373 (2015).

15. B. L. Nannenga, T. Gonen, The cryo-EM method microcrystal electron diffraction (MicroED). Nature methods 16, 369-379 (2019).

16. D. Shi, B. L. Nannenga, M. G. Iadanza, T. Gonen, Three-dimensional electron crystallography of protein microcrystals. Elife 2, e01345 (2013).

17. B. L. Nannenga, D. Shi, A. G. Leslie, T. Gonen, High-resolution structure determination by continuous-rotation data collection in MicroED. Nature methods 11, 927-930 (2014).

18. H. Xu, et al., Solving a new R2lox protein structure by microcrystal electron diffraction. Sci. Adv. 5, eaax4621 (2019).

19. M. J. de la Cruz, et al., Atomic-resolution structures from fragmented protein crystals with the cryoEM method MicroED. Nat Methods 14, 399-402 (2017).

20. M. T. B. Clabbers, et al., MyD88 TIR domain higher-order assembly interactions revealed by microcrystal electron diffraction and serial femtosecond crystallography. Nat Commun 12, 2578 (2021). 
22. E. V. Beale, et al., A Workflow for Protein Structure Determination From Thin Crystal Lamella by Micro-Electron Diffraction. Front. Mol. Biosci. 7, 179 (2020).

23. M. W. Martynowycz, W. Zhao, J. Hattne, G. J. Jensen, T. Gonen, Collection of Continuous Rotation MicroED Data from Ion Beam-Milled Crystals of Any Size. Structure 27, 545-548.e2 (2019).

24. H. Zhou, Z. Luo, X. Li, Using focus ion beam to prepare crystal lamella for electron diffraction. Journal of Structural Biology 205, 59-64 (2019).

25. X. Li, S. Zhang, J. Zhang, F. Sun, In situ protein micro-crystal fabrication by cryo-FIB for electron diffraction. Biophys Rep 4, 339-347 (2018).

26. V. Polovinkin, et al., Demonstration of electron diffraction from membrane protein crystals grown in a lipidic mesophase after lamella preparation by focused ion beam milling at cryogenic temperatures. J Appl Crystallogr 53 (2020).

27. M. Marko, C. Hsieh, R. Schalek, J. Frank, C. Mannella, Focused-ion-beam thinning of frozenhydrated biological specimens for cryo-electron microscopy. Nature methods 4, 215-217 (2007).

28. F. R. Wagner, et al., Preparing samples from whole cells using focused-ion-beam milling for cryoelectron tomography. Nat Protoc 15, 2041-2070 (2020).

29. M. W. Martynowycz, W. Zhao, J. Hattne, G. J. Jensen, T. Gonen, Qualitative Analyses of Polishing and Precoating FIB Milled Crystals for MicroED. Structure 27, 1594-1600.e2 (2019).

30. M. W. Martynowycz, T. Gonen, "Efficient, high-throughput ligand incorporation into protein microcrystals by on-grid soaking" (Biochemistry, 2020) https:/doi.org/10.1101/2020.05.25.115246 (August 18, 2020).

31. W. Kabsch, XDS. Acta Crystallogr D Biol Crystallogr 66, 125-132 (2010).

32. P. A. Karplus, K. Diederichs, Linking Crystallographic Model and Data Quality. Science 336, 10301033 (2012).

33. J. Hattne, et al., MicroED data collection and processing. Acta Crystallogr A Found Adv 71, 353-360 (2015).

34. K. Yonekura, T. Ishikawa, S. Maki-Yonekura, A new cryo-EM system for electron 3D crystallography by eEFD. Journal of structural biology 206, 243-253 (2019).

35. K. Yonekura, S. Maki-Yonekura, K. Namba, Quantitative Comparison of Zero-Loss and Conventional Electron Diffraction from Two-Dimensional and Thin Three-Dimensional Protein Crystals.

Biophysical Journal 82, 2784-2797 (2002).

36. T. Gonen, P. Sliz, J. Kistler, Y. Cheng, T. Walz, Aquaporin-0 membrane junctions reveal the structure of a closed water pore. Nature 429, 193-197 (2004).

37. T. Gonen, et al., Lipid-protein interactions in double-layered two-dimensional AQPO crystals. Nature 438, 633-638 (2005). 
38. M. W. Martynowycz, T. Gonen, "Studying Membrane Protein Structures by MicroED" in Structure and Function of Membrane Proteins, Methods in Molecular Biology., I. Schmidt-Krey, J. C. Gumbart, Eds. (Springer US, 2021), pp. 137-151.

39. T. Latychevskaia, J. P. Abrahams, Inelastic scattering and solvent scattering reduce dynamical diffraction in biological crystals. Acta Crystallogr B Struct Sci Cryst Eng Mater 75, 523-531 (2019).

40. M. W. Martynowycz, F. Khan, J. Hattne, J. Abramson, T. Gonen, MicroED structure of lipidembedded mammalian mitochondrial voltage-dependent anion channel. Proc Natl Acad Sci USA 117, 32380-32385 (2020).

41. M. W. Martynowycz, et al., "MicroED structure of the human adenosine receptor determined from a single nanocrystal in LCP" (Biophysics, 2020) https:/doi.org/10.1101/2020.09.27.316109 (September 30, 2020).

42. P. R. Evans, G. N. Murshudov, How good are my data and what is the resolution? Acta Crystallogr D Biol Crystallogr 69, 1204-1214 (2013).

43. W. Kabsch, Integration, scaling, space-group assignment and post-refinement. Acta Crystallogr D Biol Crystallogr 66, 133-144 (2010).

44. A. J. McCoy, et al., Phaser crystallographic software. J Appl Crystallogr 40, 658-674 (2007).

45. J. Hattne, et al., Analysis of Global and Site-Specific Radiation Damage in Cryo-EM. Structure 26, 759-766.e4 (2018).

46. P. V. Afonine, et al., Towards automated crystallographic structure refinement with phenix. refine. Acta Crystallographica Section D: Biological Crystallography 68, 352-367 (2012).

47. R. F. Egerton, S. C. Cheng, Measurement of local thickness by electron energy-loss spectroscopy. Ultramicroscopy 21, 231-244 (1987).

48. B. Feja, U. Aebi, Determination of the inelastic mean free path of electrons in vitrified ice layers for on-line thickness measurements by zero-loss imaging. Journal of Microscopy 193, 15-19 (1999).

49. J. Vörös, The Density and Refractive Index of Adsorbing Protein Layers. Biophysical Journal 87, 553-561 (2004).

50. R. Yan, et al., Simultaneous determination of sample thickness, tilt, and electron mean free path using tomographic tilt images based on Beer-Lambert law. Journal of Structural Biology 192, 287296 (2015).

51. J. Hattne, "Low-Dose Data Collection and Radiation Damage in MicroED" in CryoEM, Methods in Molecular Biology., T. Gonen, B. L. Nannenga, Eds. (Springer US, 2021), pp. 309-319.

52. M. J. Berger, J. S. Coursey, M. and Zucker, J. Chang, Stopping-power and range tables for electrons, protons, and helium ions (NIST Physics Laboratory Gaithersburg, MD, 1998). 
bioRxiv preprint doi: https://doi.org/10.1101/2021.07.02.450941; this version posted July 3, 2021. The copyright holder for this preprint (which was not certified by peer review) is the author/funder. All rights reserved. No reuse allowed without permission.

444 53. C. Nave, M. A. Hill, Will reduced radiation damage occur with very small crystals? J Synchrotron $445 \quad$ Rad 12, 299-303 (2005).

446 
Crystal 1

Crystal 21
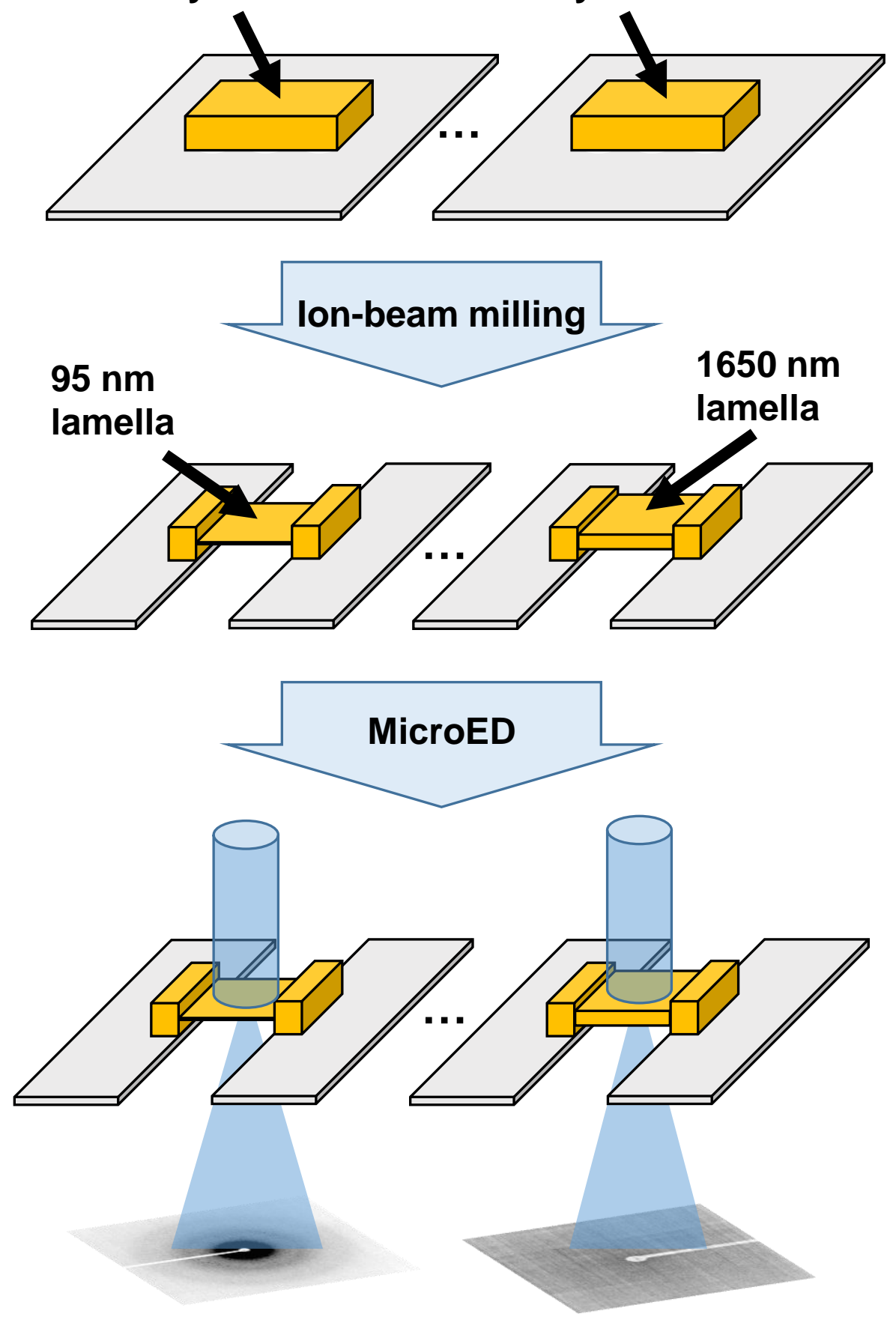

Figure 1 
120 kV
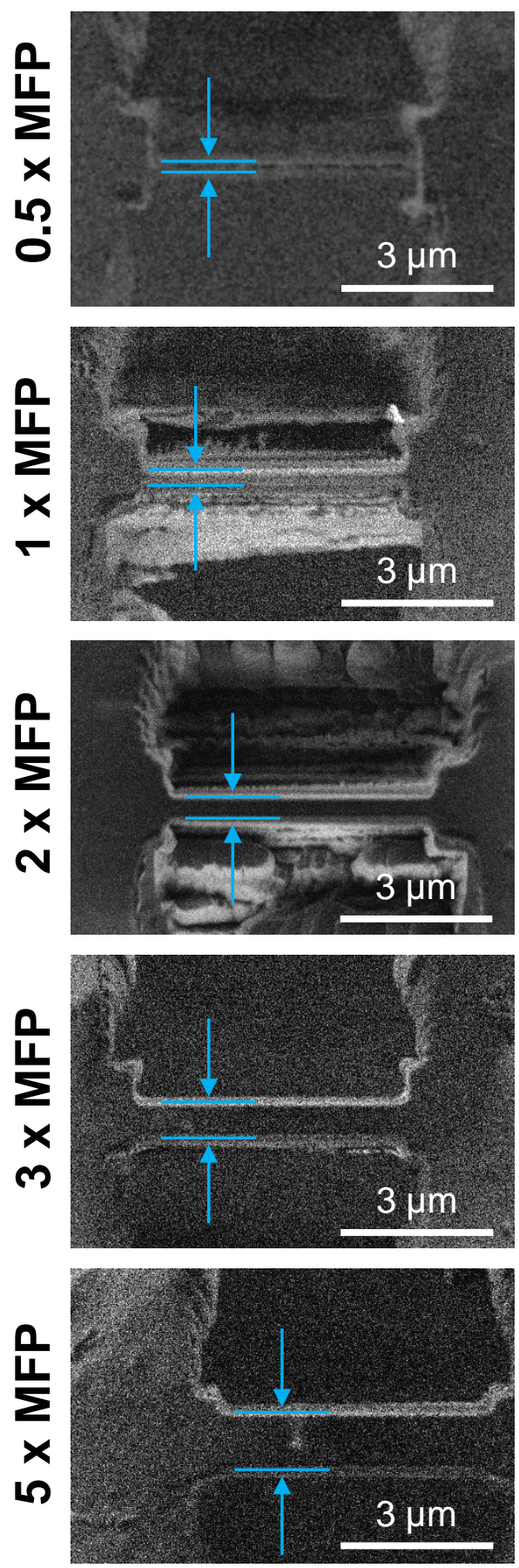

200 kV
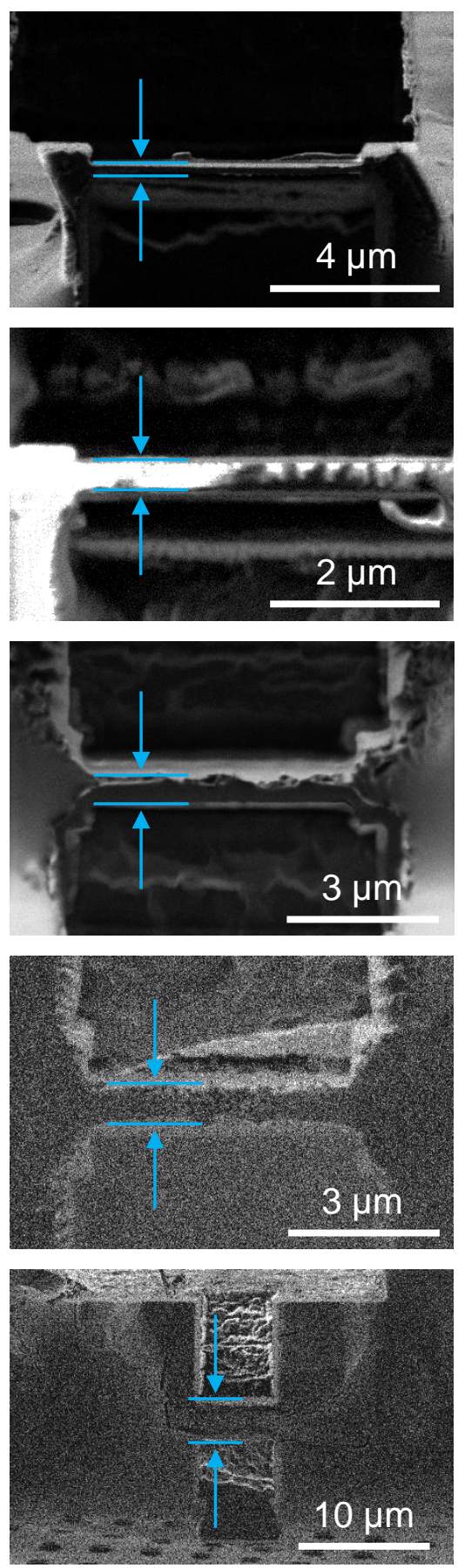

$300 \mathrm{kV}$
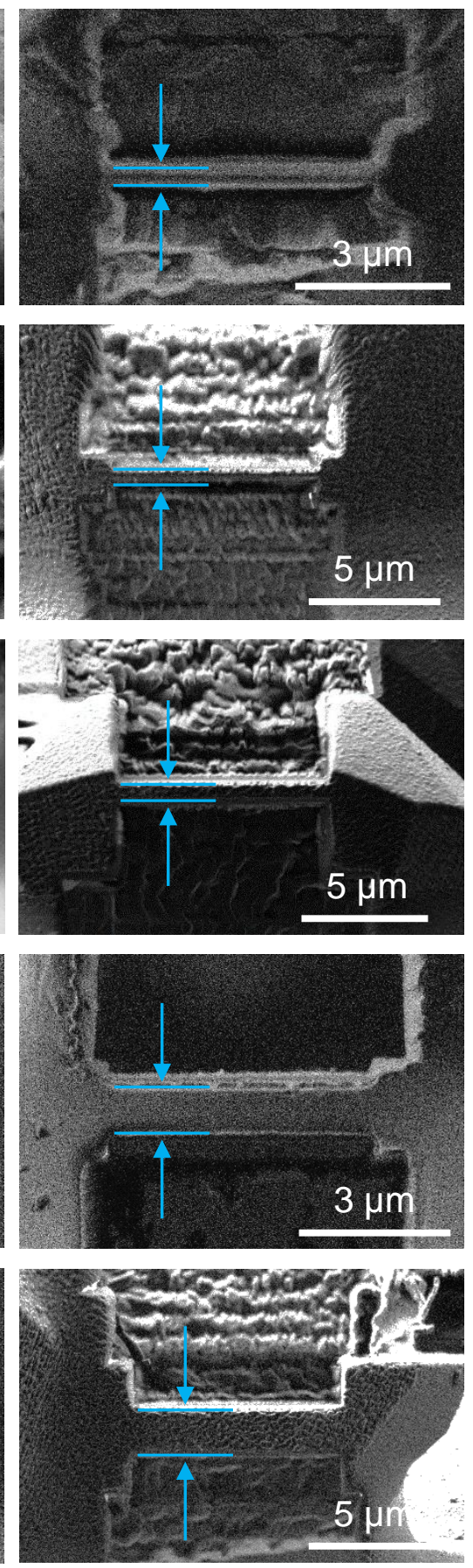

Figure 2 


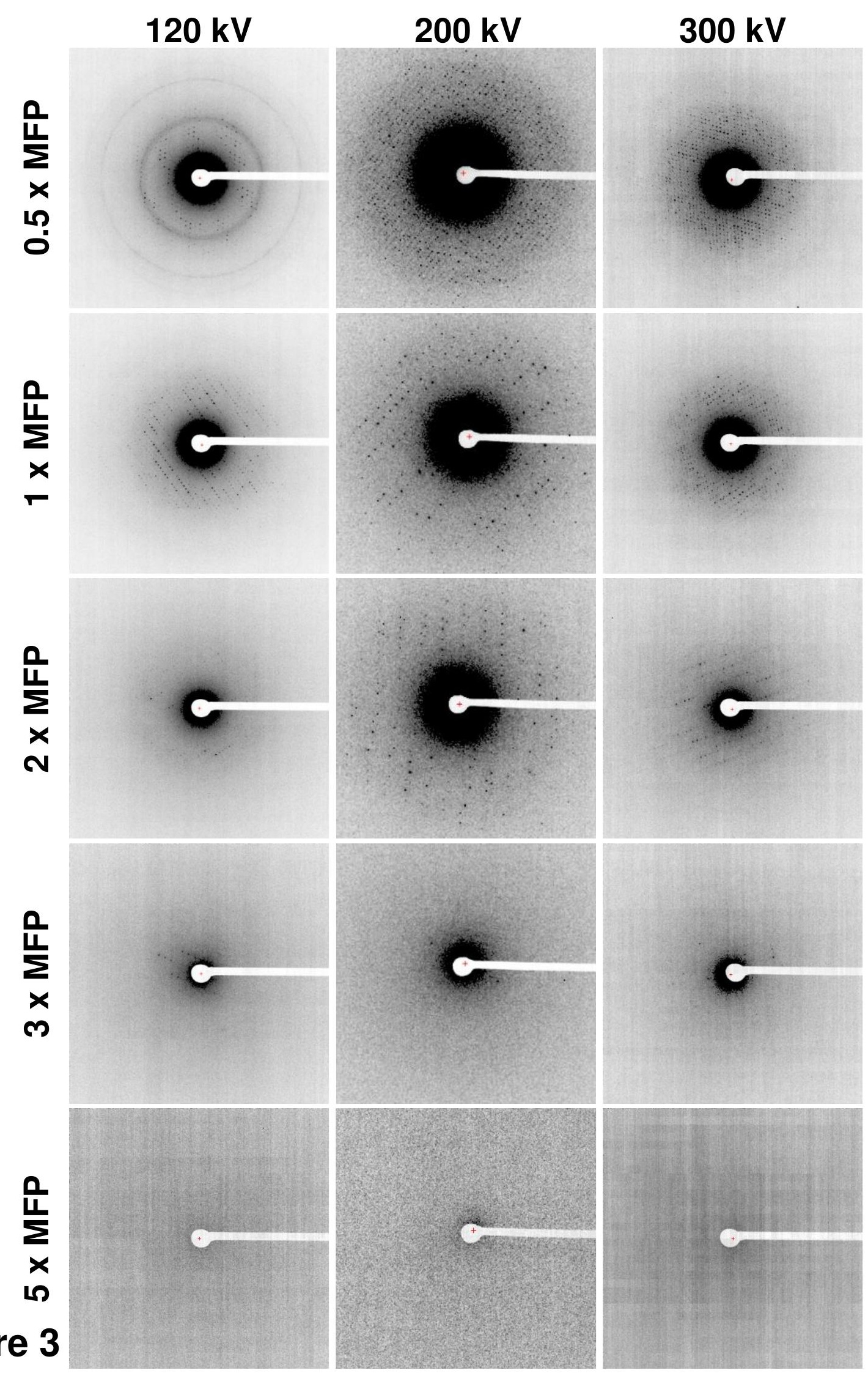



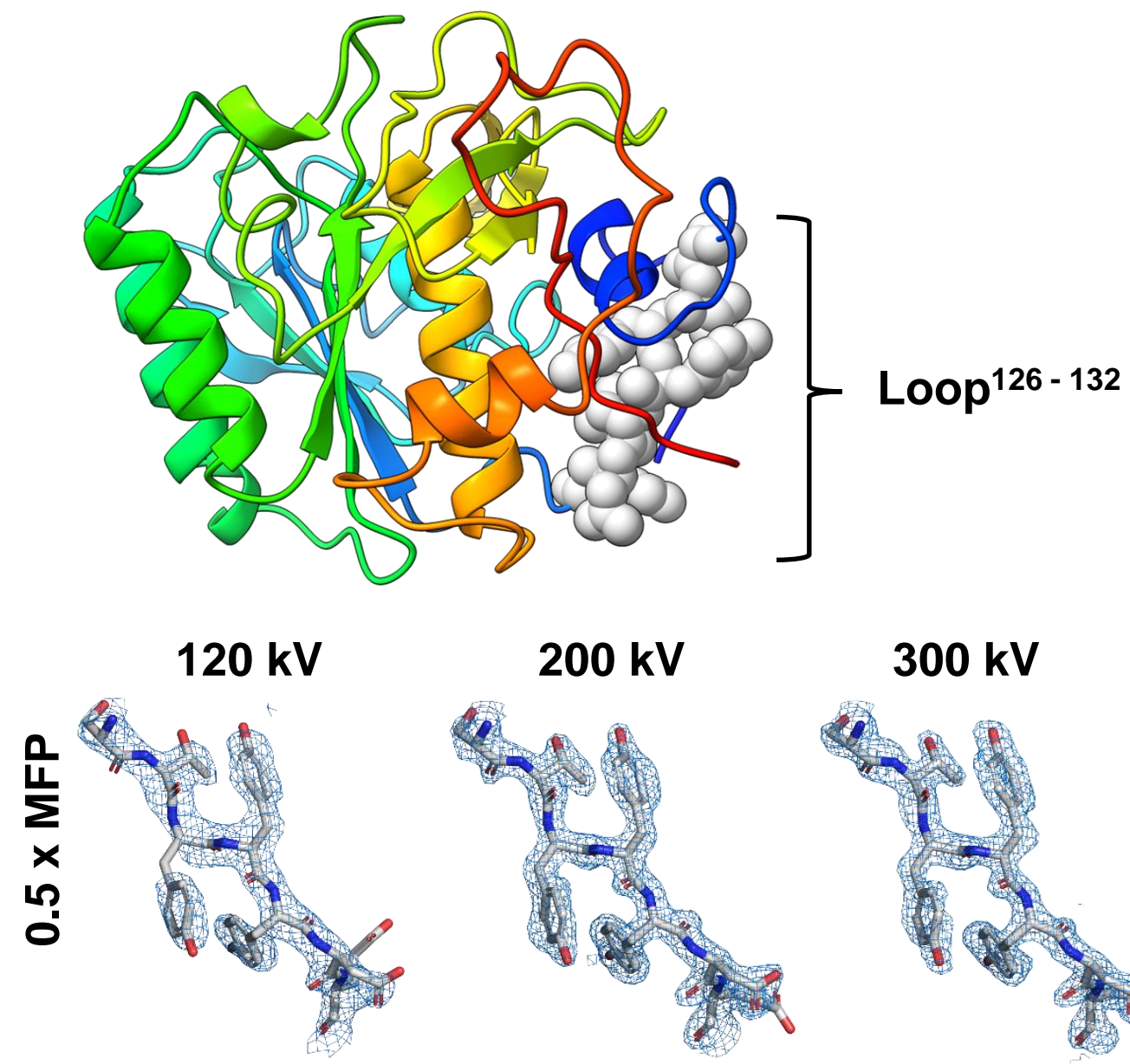

300 kV
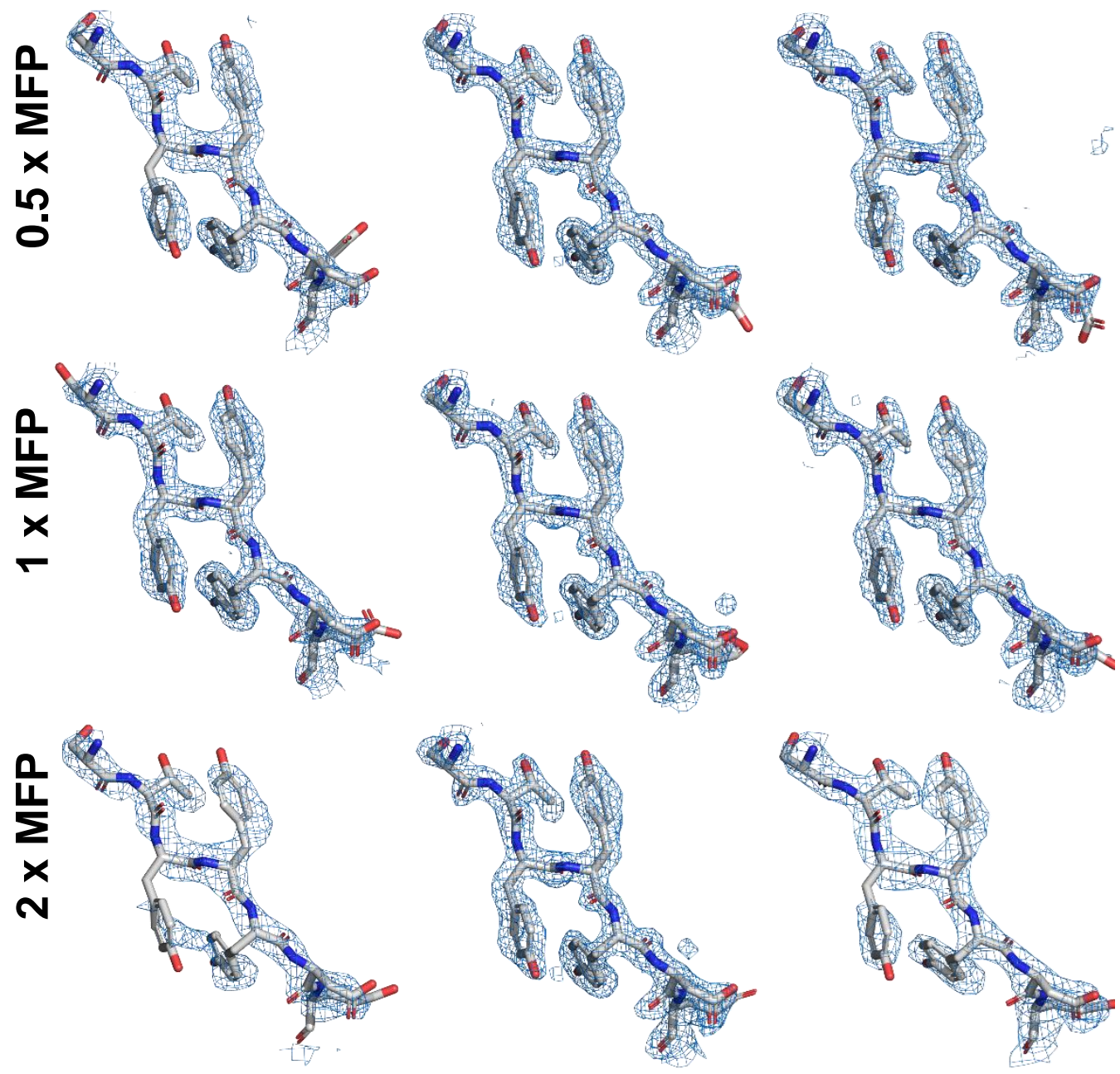

Figure 4 

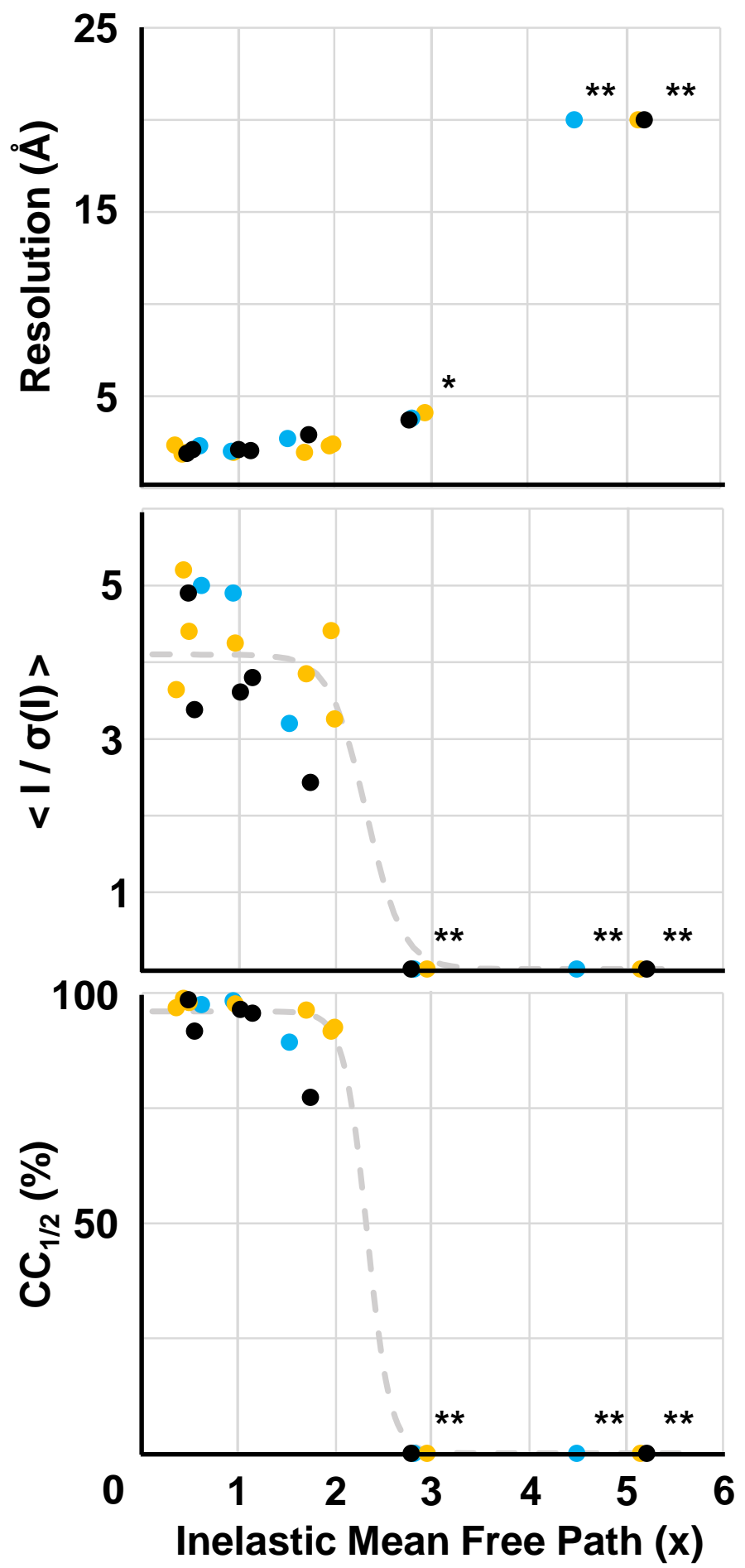

Figure 5 


\begin{tabular}{|c|c|c|c|c|c|c|c|c|c|c|}
\hline \multirow{10}{*}{$\frac{z}{2}$} & Crystal \# & 1 & 2 & 3 & 4 & 5 & & & & \\
\hline & Thickness (nm) & 130 & 200 & 325 & 600 & 960 & & & & \\
\hline & MFP $(x)$ & 0.6 & 0.9 & 1.5 & 2.8 & 4.5 & & & & \\
\hline & Resolution limit $(\AA)$ & 2.3 & 2.0 & 2.7 & $3.8^{*}$ & $20^{* *}$ & & & & \\
\hline & Completeness (\%) & 82.5 & 87.7 & 82.6 & - & - & & & & \\
\hline & R-pim (\%) & 14.9 & 12.8 & 29.6 & - & - & & & & \\
\hline & $<\mathrm{l} / \sigma(\mathrm{I})>$ & 5.0 & 4.9 & 3.2 & 0 *** & 0 *** & & & & \\
\hline & $\mathrm{CC}_{1 / 2}$ & 97.5 & 98.3 & 89.3 & $0^{* * *}$ & $0^{* * *}$ & & & & \\
\hline & R work (\%) & 22.2 & 21.5 & 24.1 & - & - & & & & \\
\hline & $\mathrm{R}$ free $(\%)$ & 26.1 & 24.3 & 28.5 & - & - & & & & \\
\hline \multirow{10}{*}{$\frac{z}{2}$} & Crystal \# & 1 & 2 & 3 & 4 & 5 & 6 & 7 & 8 & 9 \\
\hline & Thickness (nm) & 95 & 115 & 130 & 260 & 460 & 530 & 540 & 800 & 1400 \\
\hline & $\operatorname{MFP}(\mathrm{x})$ & 0.3 & 0.4 & 0.5 & 1 & 1.7 & 1.95 & 2 & 2.9 & 5.1 \\
\hline & Resolution limit $(\AA)$ & 2.35 & 1.85 & 1.95 & 1.95 & 1.95 & 2.3 & 2.4 & $4.1^{*}$ & $20^{* *}$ \\
\hline & Completeness (\%) & 86.6 & 86.0 & 96.8 & 91.1 & 91.9 & 90.0 & 78.6 & - & - \\
\hline & R-pim (\%) & 15.9 & 10.8 & 13.4 & 13.3 & 15.4 & 19.7 & 15.6 & - & - \\
\hline & $<\mathrm{I} / \sigma(\mathrm{I})>$ & 3.6 & 5.2 & 4.4 & 4.25 & 3.85 & 4.41 & 3.25 & - & - \\
\hline & CC1/2 (\%) & 96.8 & 98.8 & 97.9 & 97.6 & 96.3 & 91.7 & 92.5 & $0^{\star \star *}$ & $0^{* * *}$ \\
\hline & $\mathrm{R}$ work (\%) & 20.0 & 18.1 & 19.4 & 18.7 & 20.1 & 20.1 & 19.3 & - & - \\
\hline & $\mathrm{R}$ free $(\%)$ & 24.0 & 21.7 & 23.6 & 23.9 & 24.5 & 24.1 & 22.2 & - & - \\
\hline \multirow{10}{*}{$\begin{array}{l}\frac{1}{2} \\
\text { ? }\end{array}$} & Crystal \# & 1 & 2 & 3 & 4 & 5 & 6 & 7 & & \\
\hline & Thickness (nm) & 150 & 170 & 320 & 360 & 550 & 880 & 1650 & & \\
\hline & MFP $(x)$ & 0.47 & 0.54 & 1.0 & 1.2 & 1.7 & 2.8 & 5.2 & & \\
\hline & Resolution limit $(\AA ̊)$ & 1.9 & 2.1 & 2.1 & 2.05 & 2.9 & $3.7^{*}$ & $20^{\star *}$ & & \\
\hline & Completeness (\%) & 90.5 & 89.9 & 82.5 & 92.9 & 90 & - & - & & \\
\hline & R-pim (\%) & 11.8 & 32.4 & 14.4 & 12.8 & 23.1 & - & - & & \\
\hline & $<\mathrm{l} / \sigma(\mathrm{I})>$ & 4.9 & 3.38 & 3.61 & 3.8 & 2.43 & - & - & & \\
\hline & CC1/2 (\%) & 98.5 & 91.7 & 96.4 & 95.6 & 77.3 & $0^{* \star *}$ & $0^{\star * \star}$ & & \\
\hline & R work (\%) & 19.17 & 27.25 & 19.85 & 19.14 & 23.8 & - & - & & \\
\hline & $\mathrm{R}$ free $(\%)$ & 22.64 & 33.20 & 24.46 & 23.64 & 27.45 & - & - & & \\
\hline
\end{tabular}

*Value approximated from diffraction images that could not be automatically processed

**Best possible value taken from beamstop cut off

*** Value presumed from inability to integrate datasets 


\title{
Supplementary Appendix for:
}

\section{Benchmarking ideal sample thickness in cryo-EM using MicroED}

\author{
Michael W. Martynowycz ${ }^{1,2}$, Max T.B. Clabbers ${ }^{2}$, Johan Unge ${ }^{2}$, Johan Hattne ${ }^{1,2}$ and \\ Tamir Gonen ${ }^{1,2,3 *}$
}

${ }^{1}$ Howard Hughes Medical Institute, University of California Los Angeles, Los Angeles CA 90095

2 Department of Biological Chemistry, University of California Los Angeles, Los Angeles CA 90095

${ }^{3}$ Department of Physiology, University of California Los Angeles, Los Angeles CA 90095

To whom correspondence should be sent: tgonen@g.ucla.edu

Supplementary Figure 1. Data from a $130 \mathrm{~nm}$ lamella of proteinase K collected at $120 \mathrm{kV}$. (Top) Image in the FIB directly after the milling finished. (Bottom Left) Single frame of MicroED data (Bottom Right) Maximum projection of the entire MicroED dataset onto a single frame.

Supplementary Figure 2. Structure of Proteinase $\mathrm{K}$ determined from a $130 \mathrm{~nm}$ lamella at 120 $\mathrm{kV}$. (Left Top) Cartoon structure. (Left Bottom) $2 \mathrm{mF}_{\mathrm{o}}-\mathrm{F}_{\mathrm{c}}$ map contoured at $1.5 \sigma$ level. (Right Top) $2 \mathrm{mF}_{\mathrm{o}}-\mathrm{F}_{\mathrm{c}}$ map of the structure generated without three loop residues indicated in the structure on the right. (Right bottom) $2 \mathrm{mF}_{\mathrm{o}}-\mathrm{F}_{\mathrm{c}}$ map for the same region calculated with the missing residues.

Supplementary Figure 3. Data from a $200 \mathrm{~nm}$ lamella of proteinase K collected at $120 \mathrm{kV}$. (Top) Image in the FIB directly after the milling finished. (Bottom Left) Single frame of MicroED data (Bottom Right) Maximum projection of the entire MicroED dataset onto a single frame.

Supplementary Figure 4. Structure of Proteinase K determined from a $200 \mathrm{~nm}$ lamella at 120 $\mathrm{kV}$. (Left Top) Cartoon structure. (Left Bottom) $2 \mathrm{mF}_{\mathrm{o}}-\mathrm{F}_{\mathrm{c}}$ map contoured at $1.5 \sigma$ level. (Right Top) $2 m F_{o}-F_{c}$ map of the structure generated without three loop residues indicated in the structure on the right. (Right bottom) $2 \mathrm{mF}_{\mathrm{o}}-\mathrm{F}_{\mathrm{c}}$ map for the same region calculated with the missing residues. 
Supplementary Figure 5. Data from a $325 \mathrm{~nm}$ lamella of proteinase K collected at $120 \mathrm{kV}$. (Top) Image in the FIB directly after the milling finished. (Bottom Left) Single frame of MicroED data (Bottom Right) Maximum projection of the entire MicroED dataset onto a single frame.

Supplementary Figure 6. Structure of Proteinase K determined from a $325 \mathrm{~nm}$ lamella at 120 $\mathrm{kV}$. (Left Top) Cartoon structure. (Left Bottom) $2 \mathrm{mF}_{\mathrm{o}}-\mathrm{F}_{\mathrm{c}}$ map contoured at $1.5 \sigma$ level. (Right Top) $2 \mathrm{mF}_{\mathrm{o}}-\mathrm{F}_{\mathrm{c}}$ map of the structure generated without three loop residues indicated in the structure on the right. (Right bottom) $2 \mathrm{mF}_{0}-\mathrm{F}_{\mathrm{c}}$ map for the same region calculated with the missing residues.

Supplementary Figure 7. Data from a $600 \mathrm{~nm}$ lamella of proteinase K collected at $120 \mathrm{kV}$. (Top) Image in the FIB directly after the milling finished. (Bottom Left) Single frame of MicroED data (Bottom Right) Maximum projection of the entire MicroED dataset onto a single frame.

Supplementary Figure 8. Data from a 960 nm lamella of proteinase K collected at $120 \mathrm{kV}$. (Top) Image in the FIB directly after the milling finished. (Bottom Left) Single frame of MicroED data (Bottom Right) Maximum projection of the entire MicroED dataset onto a single frame.

Supplementary Figure 9. Data from a $95 \mathrm{~nm}$ lamella of proteinase K collected at $200 \mathrm{kV}$. (Top) Image in the FIB directly after the milling finished. (Bottom Left) Single frame of MicroED data (Bottom Right) Maximum projection of the entire MicroED dataset onto a single frame.

Supplementary Figure 10. Structure of Proteinase K determined from a $95 \mathrm{~nm}$ lamella at 200 kV. (Left Top) Cartoon structure. (Left Bottom) $2 \mathrm{mF}_{\mathrm{o}}-\mathrm{F}_{\mathrm{c}}$ map contoured at $1.5 \sigma$ level. (Right Top) $2 \mathrm{mF}_{\mathrm{o}}-\mathrm{F}_{\mathrm{c}}$ map of the structure generated without three loop residues indicated in the structure on the right. (Right bottom) $2 \mathrm{mF}_{\mathrm{o}}-\mathrm{F}_{\mathrm{c}}$ map for the same region calculated with the missing residues.

Supplementary Figure 11. Data from a $115 \mathrm{~nm}$ lamella of proteinase K collected at $200 \mathrm{kV}$. (Top) Image in the FIB directly after the milling finished. (Bottom Left) Single frame of MicroED data (Bottom Right) Maximum projection of the entire MicroED dataset onto a single frame.

Supplementary Figure 12. Structure of Proteinase K determined from a $115 \mathrm{~nm}$ lamella at 200 kV. (Left Top) Cartoon structure. (Left Bottom) $2 \mathrm{mF}_{\mathrm{o}}-\mathrm{F}_{\mathrm{c}}$ map contoured at $1.5 \sigma$ level. (Right Top) $2 \mathrm{mF}_{\mathrm{o}}-\mathrm{F}_{\mathrm{c}}$ map of the structure generated without three loop residues indicated in the structure on the right. (Right bottom) $2 \mathrm{mF}_{\mathrm{o}}-\mathrm{F}_{\mathrm{c}}$ map for the same region calculated with the missing residues.

Supplementary Figure 13. Data from a $130 \mathrm{~nm}$ lamella of proteinase K collected at $200 \mathrm{kV}$. (Top) Image in the FIB directly after the milling finished. (Bottom Left) Single frame of MicroED data (Bottom Right) Maximum projection of the entire MicroED dataset onto a single frame.

Supplementary Figure 14. Structure of Proteinase K determined from a $130 \mathrm{~nm}$ lamella at 200 kV. (Left Top) Cartoon structure. (Left Bottom) $2 \mathrm{mF}_{\mathrm{o}}-\mathrm{F}_{\mathrm{c}}$ map contoured at $1.5 \sigma$ level. (Right Top) $2 \mathrm{mF}_{\mathrm{o}}-\mathrm{F}_{\mathrm{c}}$ map of the structure generated without three loop residues indicated in the structure on the right. (Right bottom) $2 \mathrm{mF}_{\mathrm{o}}-\mathrm{F}_{\mathrm{c}}$ map for the same region calculated with the missing residues.

Supplementary Figure 15. Data from a $260 \mathrm{~nm}$ lamella of proteinase K collected at $200 \mathrm{kV}$. (Top) Image in the FIB directly after the milling finished. (Bottom Left) Single frame of MicroED data (Bottom Right) Maximum projection of the entire MicroED dataset onto a single frame.

Supplementary Figure 16. Structure of Proteinase K determined from a $260 \mathrm{~nm}$ lamella at 200 kV. (Left Top) Cartoon structure. (Left Bottom) $2 \mathrm{mF}_{\mathrm{o}}-\mathrm{F}_{\mathrm{c}}$ map contoured at $1.5 \sigma$ level. (Right Top) $2 \mathrm{mF}_{\mathrm{o}}-\mathrm{F}_{\mathrm{c}}$ map of the structure generated without three loop residues indicated in the structure on the right. (Right bottom) $2 \mathrm{mF}_{\mathrm{o}}-\mathrm{F}_{\mathrm{c}}$ map for the same region calculated with the missing residues. 
Supplementary Figure 17. Data from a $460 \mathrm{~nm}$ lamella of proteinase K collected at $200 \mathrm{kV}$. (Top) Image in the FIB directly after the milling finished. (Bottom Left) Single frame of MicroED data (Bottom Right) Maximum projection of the entire MicroED dataset onto a single frame.

Supplementary Figure 18. Structure of Proteinase K determined from a $460 \mathrm{~nm}$ lamella at 200 kV. (Left Top) Cartoon structure. (Left Bottom) $2 \mathrm{mF}_{\mathrm{o}}-\mathrm{F}_{\mathrm{c}}$ map contoured at $1.5 \sigma$ level. (Right Top) $2 \mathrm{mF}_{\mathrm{o}}-\mathrm{F}_{\mathrm{c}}$ map of the structure generated without three loop residues indicated in the structure on the right. (Right bottom) $2 \mathrm{mF}_{0}-\mathrm{F}_{\mathrm{c}}$ map for the same region calculated with the missing residues.

Supplementary Figure 19. Data from a $530 \mathrm{~nm}$ lamella of proteinase K collected at $200 \mathrm{kV}$. (Top) Image in the FIB directly after the milling finished. (Bottom Left) Single frame of MicroED data (Bottom Right) Maximum projection of the entire MicroED dataset onto a single frame.

Supplementary Figure 20. Structure of Proteinase K determined from a $530 \mathrm{~nm}$ lamella at 200 kV. (Left Top) Cartoon structure. (Left Bottom) $2 \mathrm{mF}_{\mathrm{o}}-\mathrm{F}_{\mathrm{c}}$ map contoured at $1.5 \sigma$ level. (Right Top) $2 \mathrm{mF}_{\mathrm{o}}-\mathrm{F}_{\mathrm{c}}$ map of the structure generated without three loop residues indicated in the structure on the right. (Right bottom) $2 \mathrm{mF}_{\mathrm{o}}-\mathrm{F}_{\mathrm{c}}$ map for the same region calculated with the missing residues.

Supplementary Figure 21. Data from a $540 \mathrm{~nm}$ lamella of proteinase K collected at $200 \mathrm{kV}$. (Top) Image in the FIB directly after the milling finished. (Bottom Left) Single frame of MicroED data (Bottom Right) Maximum projection of the entire MicroED dataset onto a single frame.

Supplementary Figure 22. Structure of Proteinase K determined from a $540 \mathrm{~nm}$ lamella at 200 kV. (Left Top) Cartoon structure. (Left Bottom) $2 \mathrm{mF}_{\mathrm{o}}-\mathrm{F}_{\mathrm{c}}$ map contoured at $1.5 \sigma$ level. (Right Top) $2 \mathrm{mF}_{\mathrm{o}}-\mathrm{F}_{\mathrm{c}}$ map of the structure generated without three loop residues indicated in the structure on the right. (Right bottom) $2 \mathrm{mF}_{\mathrm{o}}-\mathrm{F}_{\mathrm{c}}$ map for the same region calculated with the missing residues.

Supplementary Figure 23. Data from an $800 \mathrm{~nm}$ lamella of proteinase K collected at $200 \mathrm{kV}$. (Top) Image in the FIB directly after the milling finished. (Bottom Left) Single frame of MicroED data (Bottom Right) Maximum projection of the entire MicroED dataset onto a single frame.

Supplementary Figure 24. Data from a $1400 \mathrm{~nm}$ lamella of proteinase K collected at $200 \mathrm{kV}$. (Top) Image in the FIB directly after the milling finished. (Bottom Left) Single frame of MicroED data (Bottom Right) Maximum projection of the entire MicroED dataset onto a single frame.

Supplementary Figure 25. Data from a $150 \mathrm{~nm}$ lamella of proteinase K collected at $300 \mathrm{kV}$. (Top) Image in the FIB directly after the milling finished. (Bottom Left) Single frame of MicroED data (Bottom Right) Maximum projection of the entire MicroED dataset onto a single frame.

Supplementary Figure 26. Structure of Proteinase K determined from a $150 \mathrm{~nm}$ lamella at 300 kV. (Left Top) Cartoon structure. (Left Bottom) $2 \mathrm{mF}_{\mathrm{o}}-\mathrm{F}_{\mathrm{c}}$ map contoured at $1.5 \sigma$ level. (Right Top) $2 \mathrm{mF}_{\mathrm{o}}-\mathrm{F}_{\mathrm{c}}$ map of the structure generated without three loop residues indicated in the structure on the right. (Right bottom) $2 \mathrm{mF}_{\mathrm{o}}-\mathrm{F}_{\mathrm{c}}$ map for the same region calculated with the missing residues.

Supplementary Figure 27. Data from a $170 \mathrm{~nm}$ lamella of proteinase K collected at $300 \mathrm{kV}$. (Top) Image in the FIB directly after the milling finished. (Bottom Left) Single frame of MicroED data (Bottom Right) Maximum projection of the entire MicroED dataset onto a single frame.

Supplementary Figure 28. Structure of Proteinase K determined from a $170 \mathrm{~nm}$ lamella at 300 kV. (Left Top) Cartoon structure. (Left Bottom) $2 \mathrm{mF}_{\mathrm{o}}-\mathrm{F}_{\mathrm{c}}$ map contoured at $1.5 \sigma$ level. (Right Top) $2 \mathrm{mF}_{\mathrm{o}}-\mathrm{F}_{\mathrm{c}}$ map of the structure generated without three loop residues indicated in the structure on the right. (Right bottom) $2 \mathrm{mF}_{\mathrm{o}}-\mathrm{F}_{\mathrm{c}}$ map for the same region calculated with the missing residues. 
Supplementary Figure 29. Data from a $320 \mathrm{~nm}$ lamella of proteinase K collected at $300 \mathrm{kV}$. (Top) Image in the FIB directly after the milling finished. (Bottom Left) Single frame of MicroED data (Bottom Right) Maximum projection of the entire MicroED dataset onto a single frame.

Supplementary Figure 30. Structure of Proteinase K determined from a $320 \mathrm{~nm}$ lamella at 300 kV. (Left Top) Cartoon structure. (Left Bottom) $2 \mathrm{mF}_{\mathrm{o}}-\mathrm{F}_{\mathrm{c}}$ map contoured at $1.5 \sigma$ level. (Right Top) $2 \mathrm{mF}_{\mathrm{o}}-\mathrm{F}_{\mathrm{c}}$ map of the structure generated without three loop residues indicated in the structure on the right. (Right bottom) $2 \mathrm{mF}_{0}-\mathrm{F}_{\mathrm{c}}$ map for the same region calculated with the missing residues.

Supplementary Figure 31. Data from a $360 \mathrm{~nm}$ lamella of proteinase $\mathrm{K}$ collected at $300 \mathrm{kV}$. (Top) Image in the FIB directly after the milling finished. (Bottom Left) Single frame of MicroED data (Bottom Right) Maximum projection of the entire MicroED dataset onto a single frame.

Supplementary Figure 32. Structure of Proteinase K determined from a $360 \mathrm{~nm}$ lamella at 300 kV. (Left Top) Cartoon structure. (Left Bottom) $2 \mathrm{mF}_{\mathrm{o}}-\mathrm{F}_{\mathrm{c}}$ map contoured at $1.5 \sigma$ level. (Right Top) $2 \mathrm{mF}_{\mathrm{o}}-\mathrm{F}_{\mathrm{c}}$ map of the structure generated without three loop residues indicated in the structure on the right. (Right bottom) $2 \mathrm{mF}_{\mathrm{o}}-\mathrm{F}_{\mathrm{c}}$ map for the same region calculated with the missing residues.

Supplementary Figure 33. Data from a $550 \mathrm{~nm}$ lamella of proteinase K collected at $300 \mathrm{kV}$. (Top) Image in the FIB directly after the milling finished. (Bottom Left) Single frame of MicroED data (Bottom Right) Maximum projection of the entire MicroED dataset onto a single frame.

Supplementary Figure 34. Structure of Proteinase K determined from a $550 \mathrm{~nm}$ lamella at 300 kV. (Left Top) Cartoon structure. (Left Bottom) $2 \mathrm{mF}_{\mathrm{o}}-\mathrm{F}_{\mathrm{c}}$ map contoured at $1.5 \sigma$ level. (Right Top) $2 \mathrm{mF}_{\mathrm{o}}-\mathrm{F}_{\mathrm{c}}$ map of the structure generated without three loop residues indicated in the structure on the right. (Right bottom) $2 \mathrm{mF}_{\mathrm{o}}-\mathrm{F}_{\mathrm{c}}$ map for the same region calculated with the missing residues.

Supplementary Figure 35. Data from a $880 \mathrm{~nm}$ lamella of proteinase K collected at $300 \mathrm{kV}$. (Top) Image in the FIB directly after the milling finished. (Bottom Left) Single frame of MicroED data (Bottom Right) Maximum projection of the entire MicroED dataset onto a single frame.

Supplementary Figure 36. Data from a $1650 \mathrm{~nm}$ lamella of proteinase K collected at $300 \mathrm{kV}$. (Top) Image in the FIB directly after the milling finished. (Bottom Left) Single frame of MicroED data (Bottom Right) Maximum projection of the entire MicroED dataset onto a single frame.

Supplementary Figure 37. (Top) Structure of proteinase K determined by MicroED from milled lamella. (Bottom) Composite omit maps contoured at the $1.5 \sigma$ level around the loop region indicated above. Maps were generated in Phenix using electron scattering factors and all additional settings left as default.

Supplementary Table 1. MicroED data statistics for all structures and datasets in this manuscript. 


\section{Single Frame}

Maximum Projection

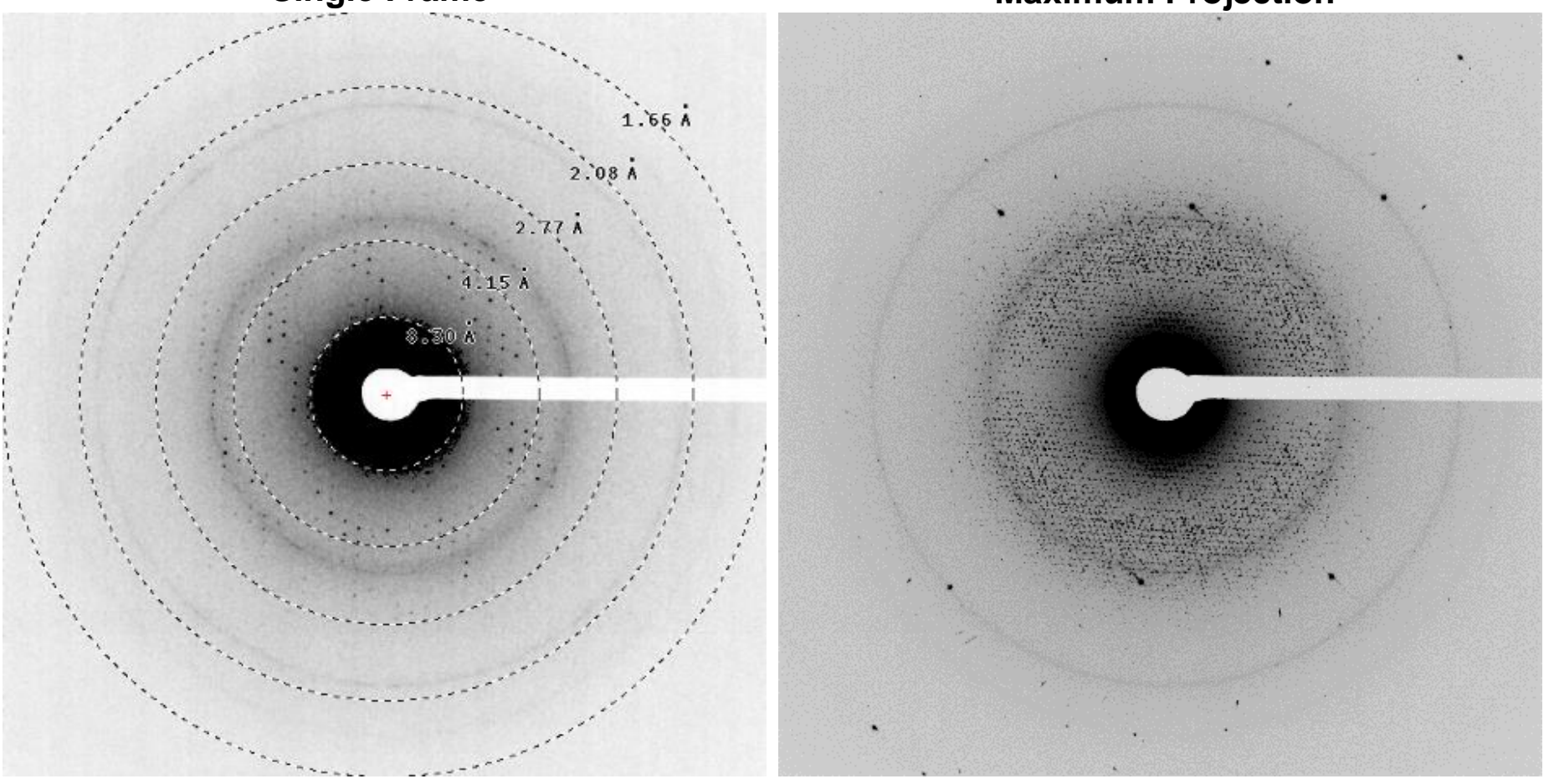

\section{SI Figure 1}




\section{$130 \mathrm{~nm}$ lamella (0.6 x MFP) $120 \mathrm{kV}$}
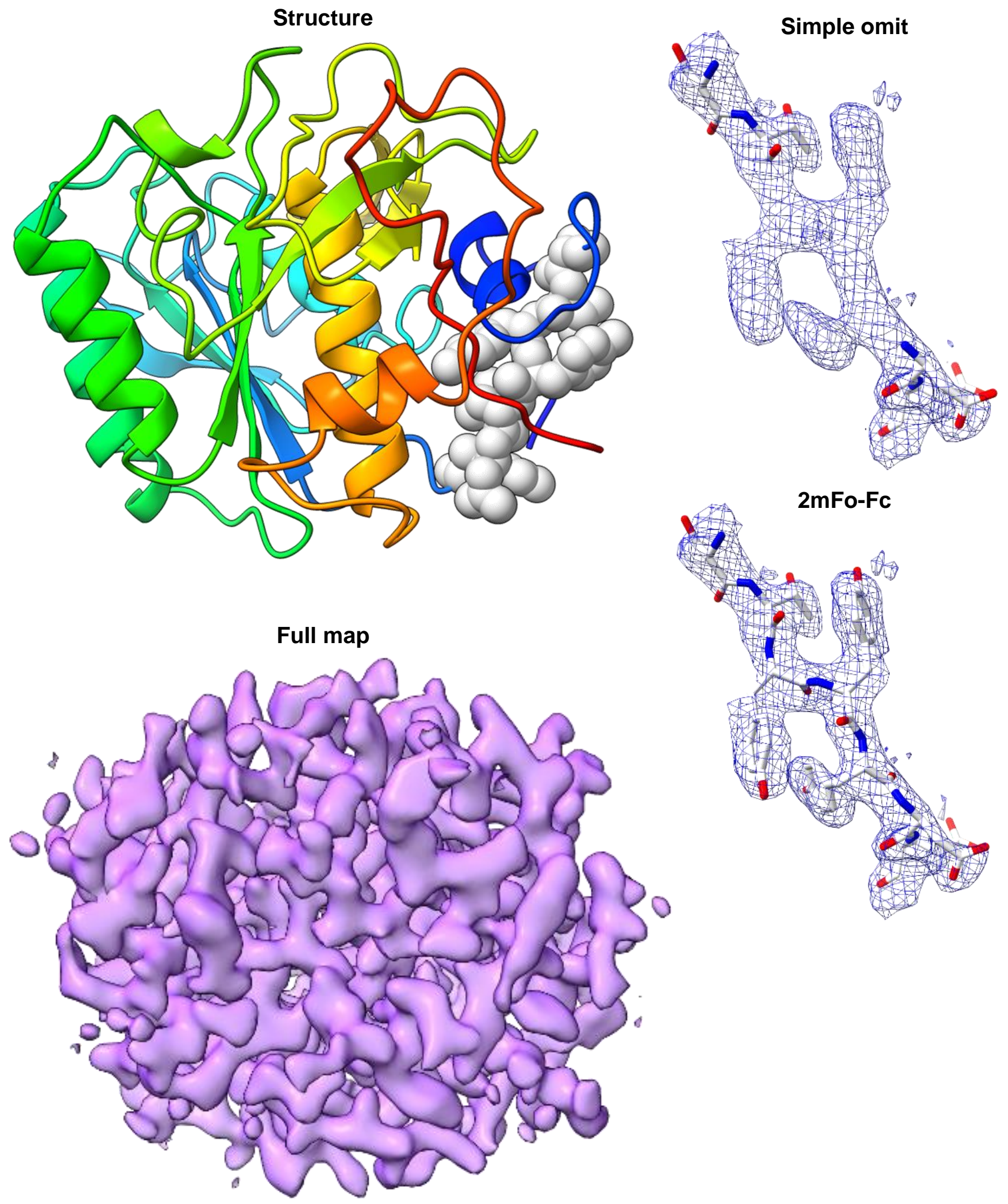

SI Figure 2 
Lamella

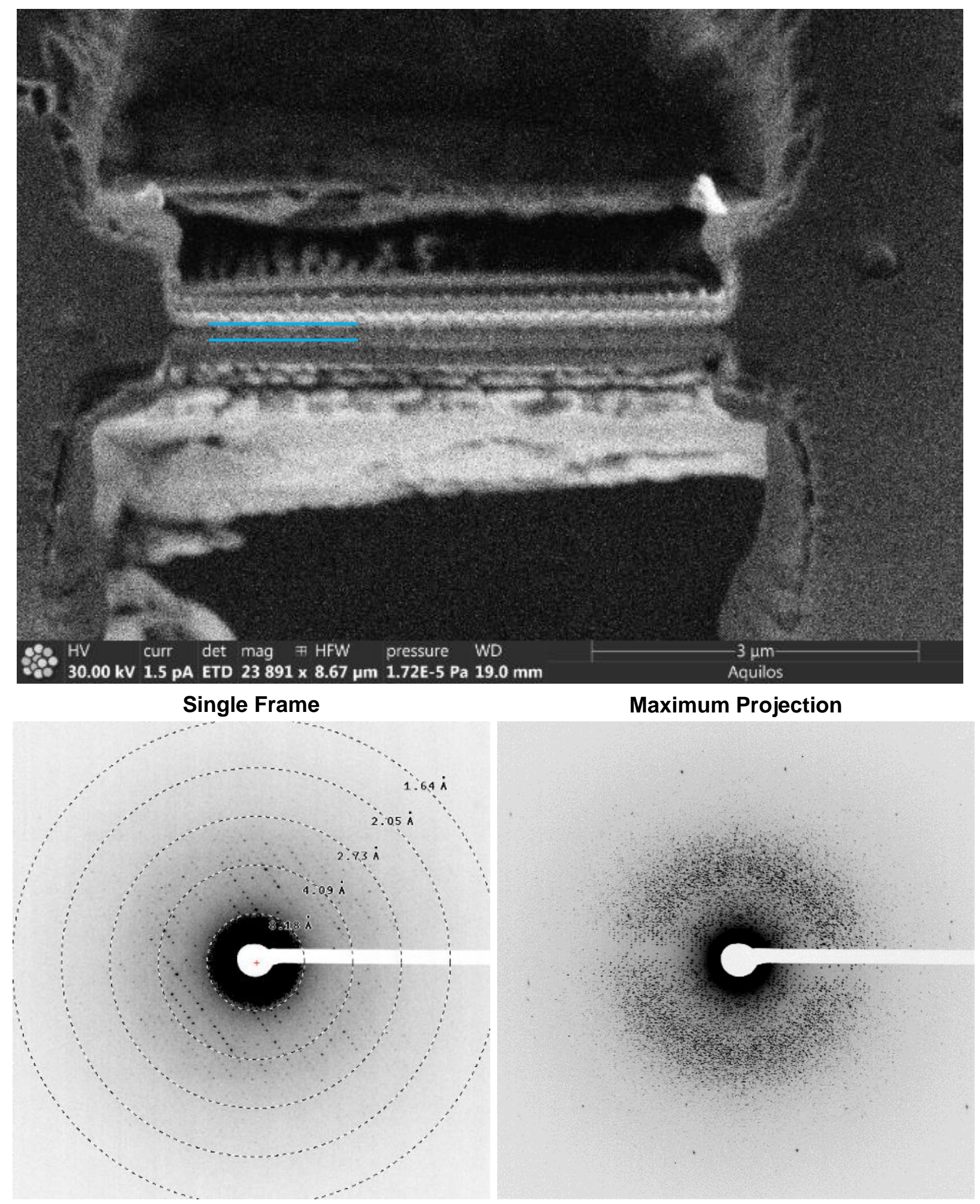

\section{SI Figure 3}




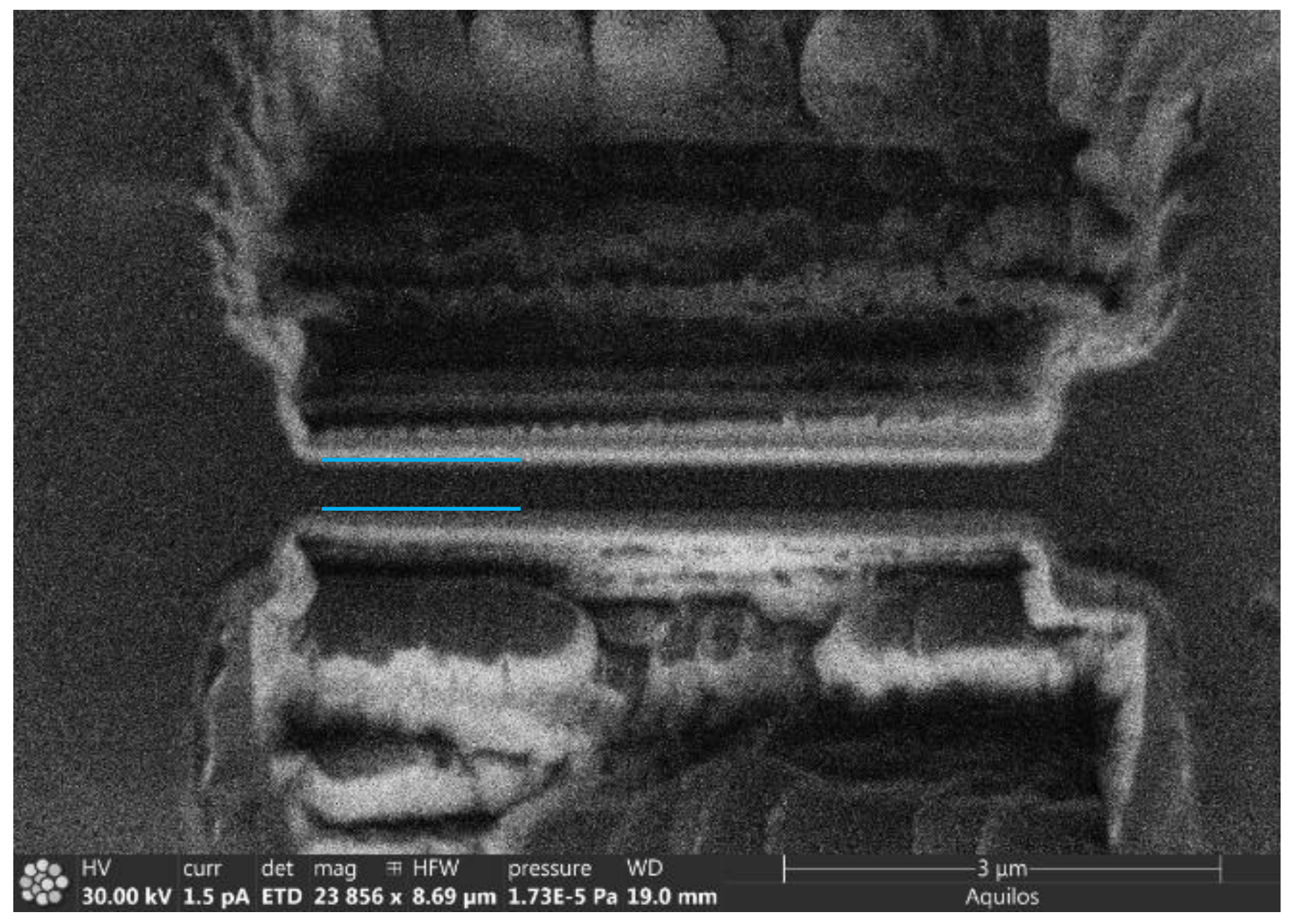

\section{Single Frame}

Maximum Projection

\section{SI Figure 5}




\section{$600 \mathrm{~nm}$ lamella (2.8 x MFP) $120 \mathrm{kV}$}

\section{Lamella}

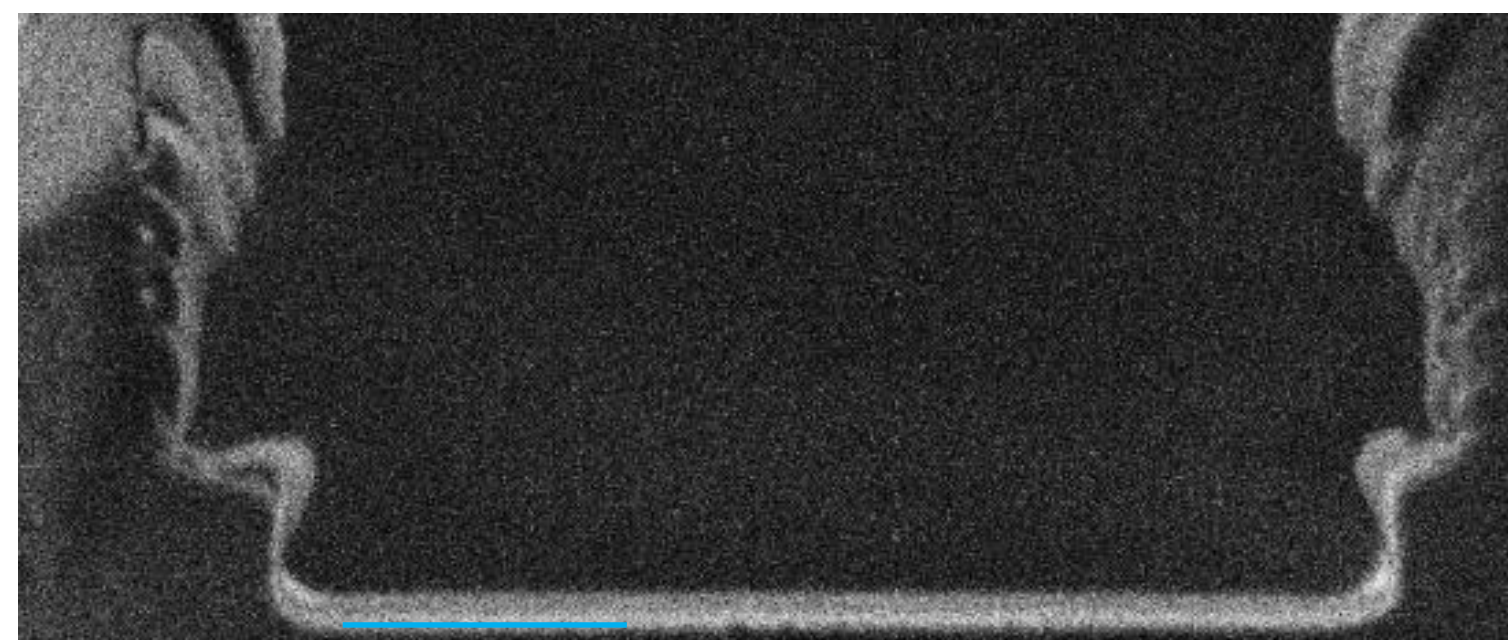

$\begin{array}{lllll}8 & \text { HV curr det mag F HFW pressure WD } \\ 30.00 \mathrm{kV} & 1.5 \text { pA ETD } 23884 \times 8.68 \mu \mathrm{m} & 1.73 \mathrm{E}-5 \mathrm{~Pa} & 19.0 \mathrm{~mm}\end{array}$

\section{Single Frame}

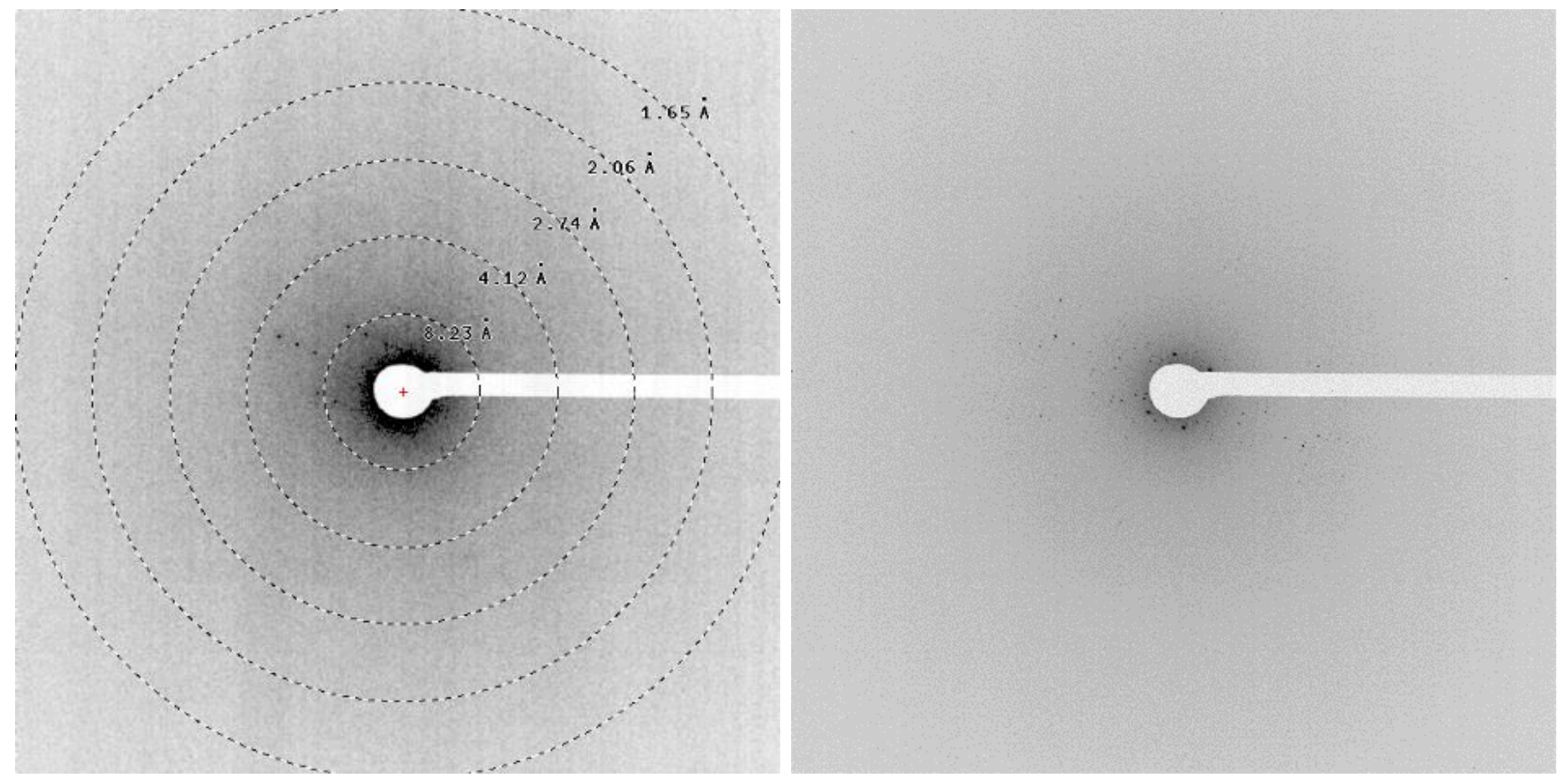

\section{SI Figure 7}



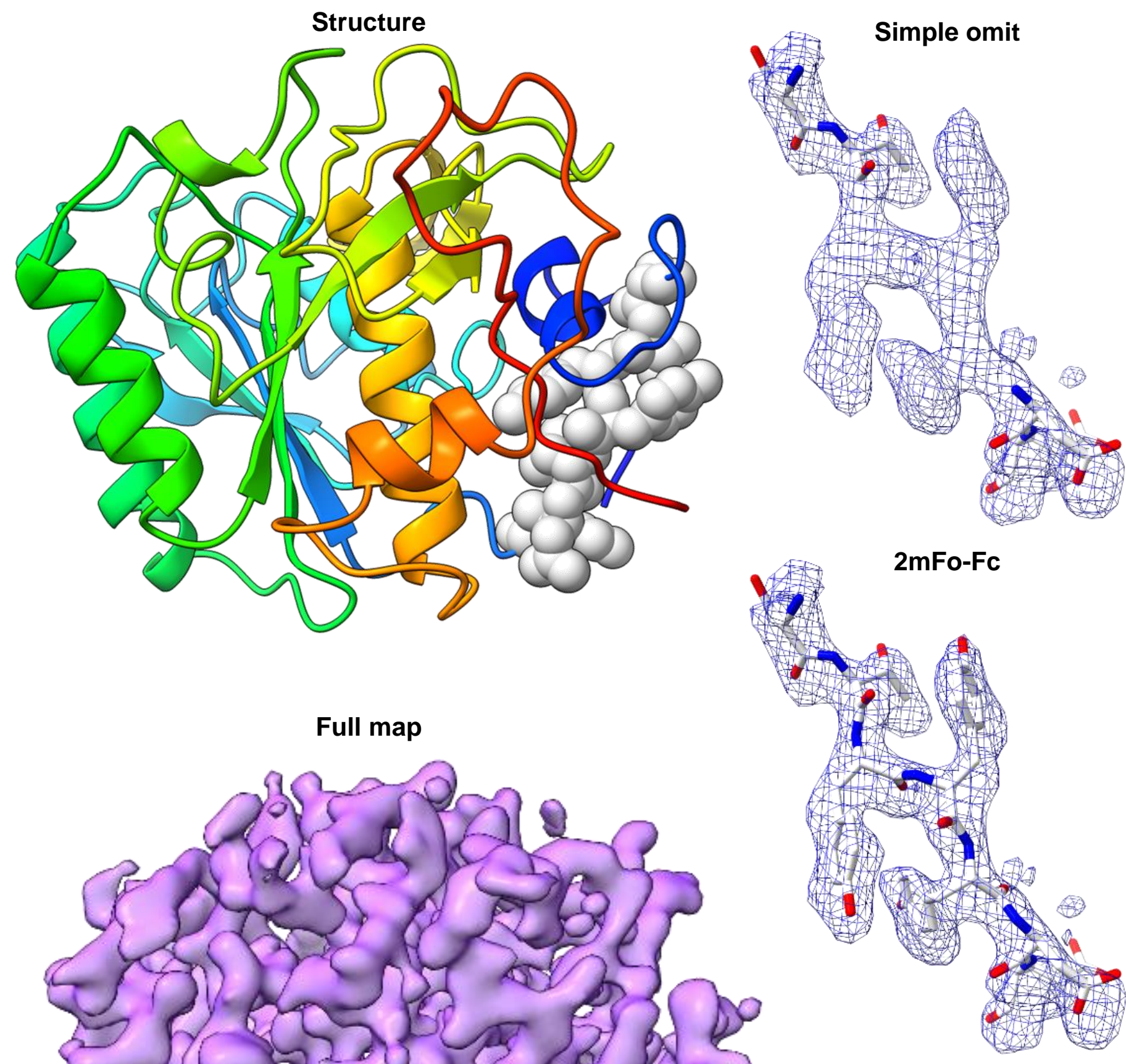

SI Figure 10

Full map

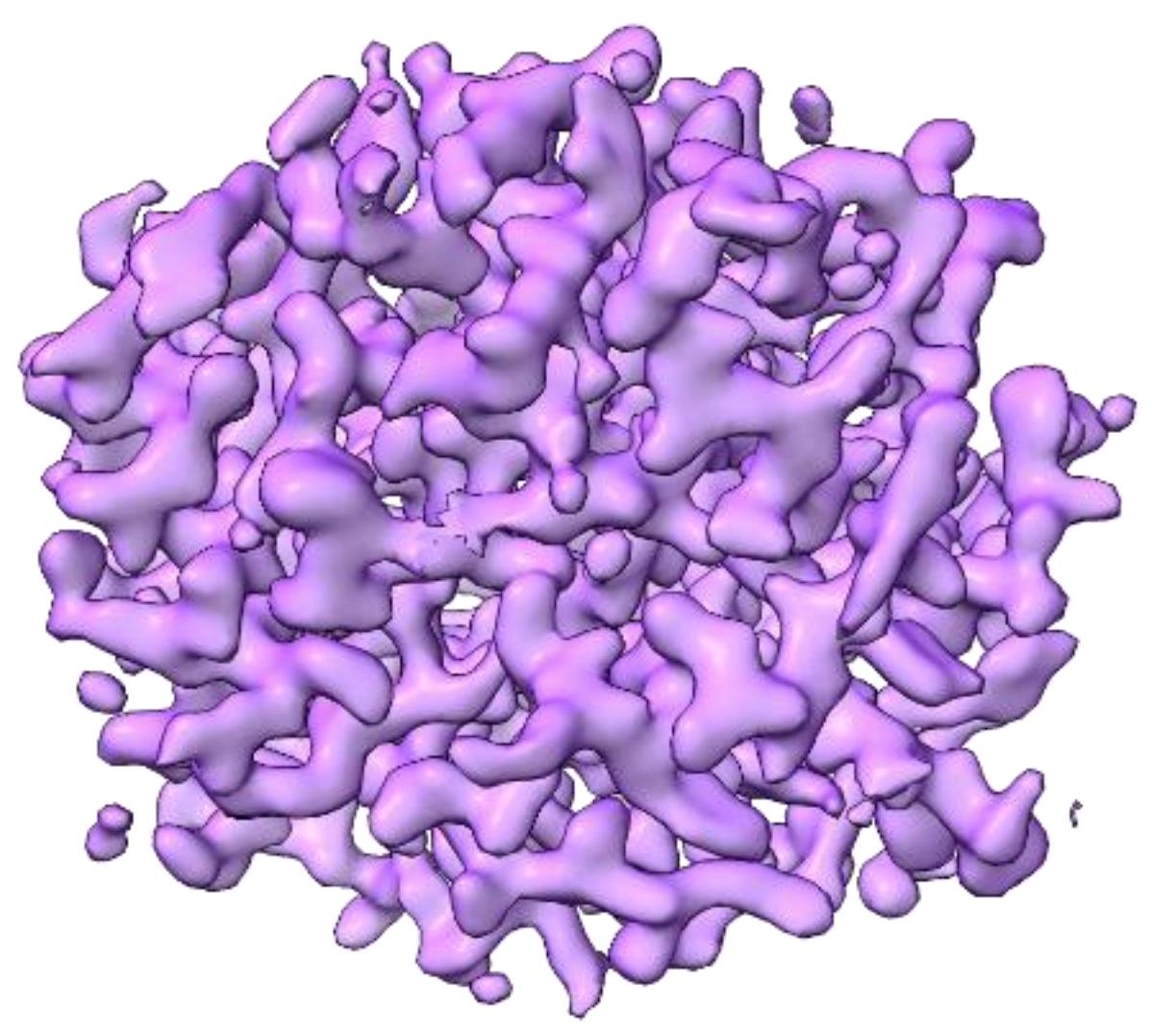




\section{$115 \mathrm{~nm}$ lamella (0.4 x MFP) $200 \mathrm{kV}$}

\section{Lamella}

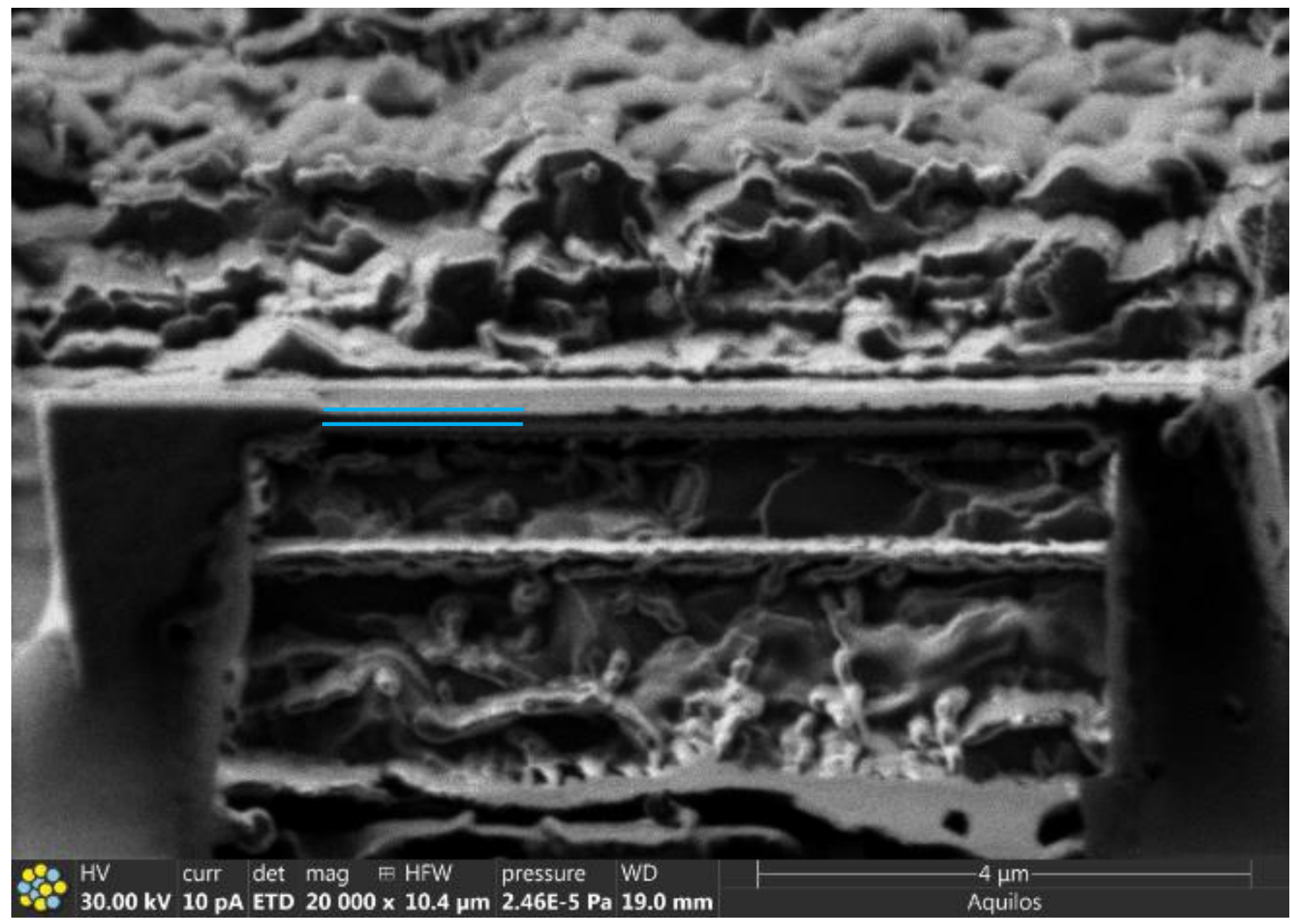

Single Frame

Maximum Projection

\section{SI Figure 11}




\section{$115 \mathrm{~nm}$ lamella (0.4 x MFP) $200 \mathrm{kV}$}
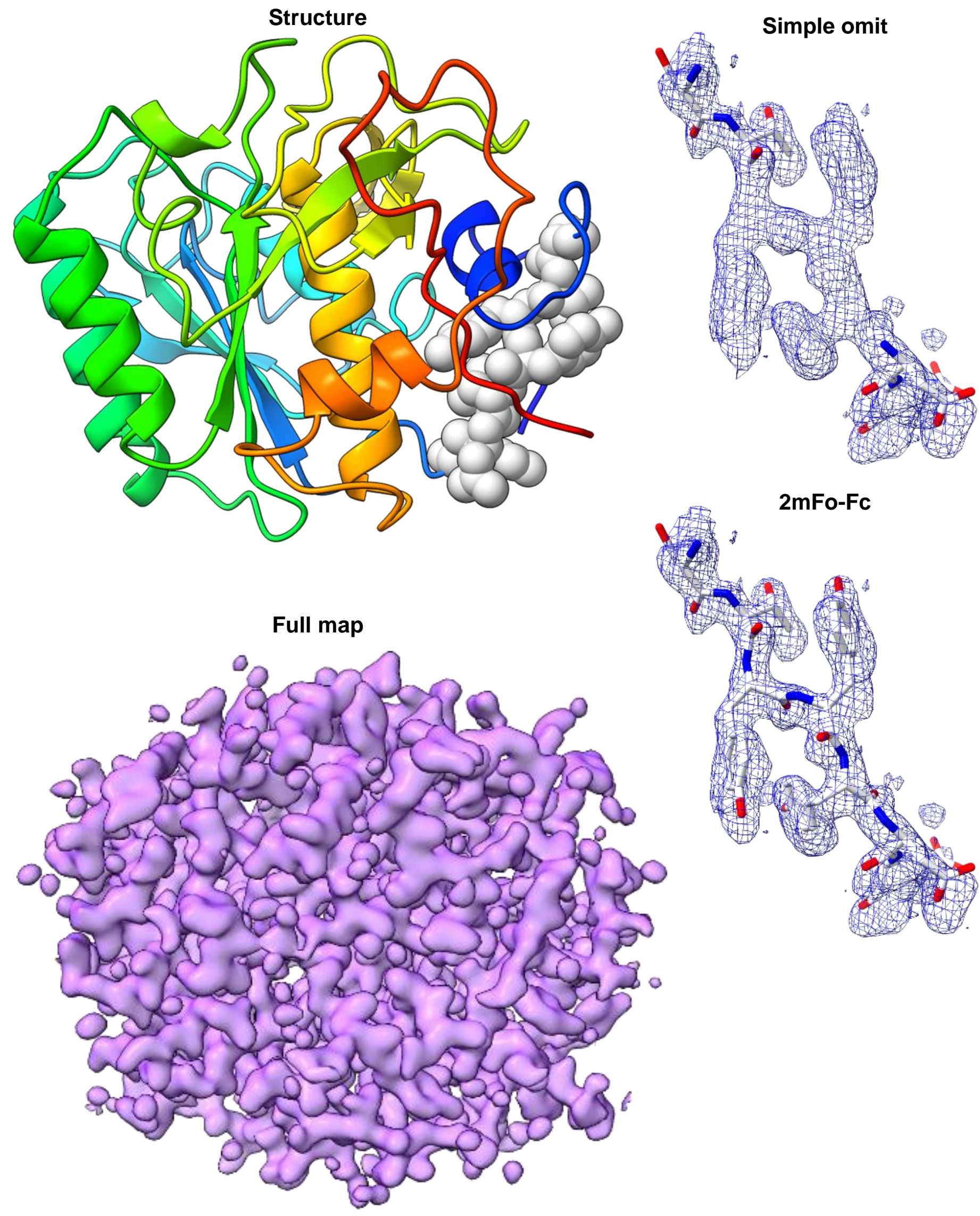

\section{SI Figure 12}




\section{$130 \mathrm{~nm}$ lamella (0.5 x MFP) $200 \mathrm{kV}$}

Lamella

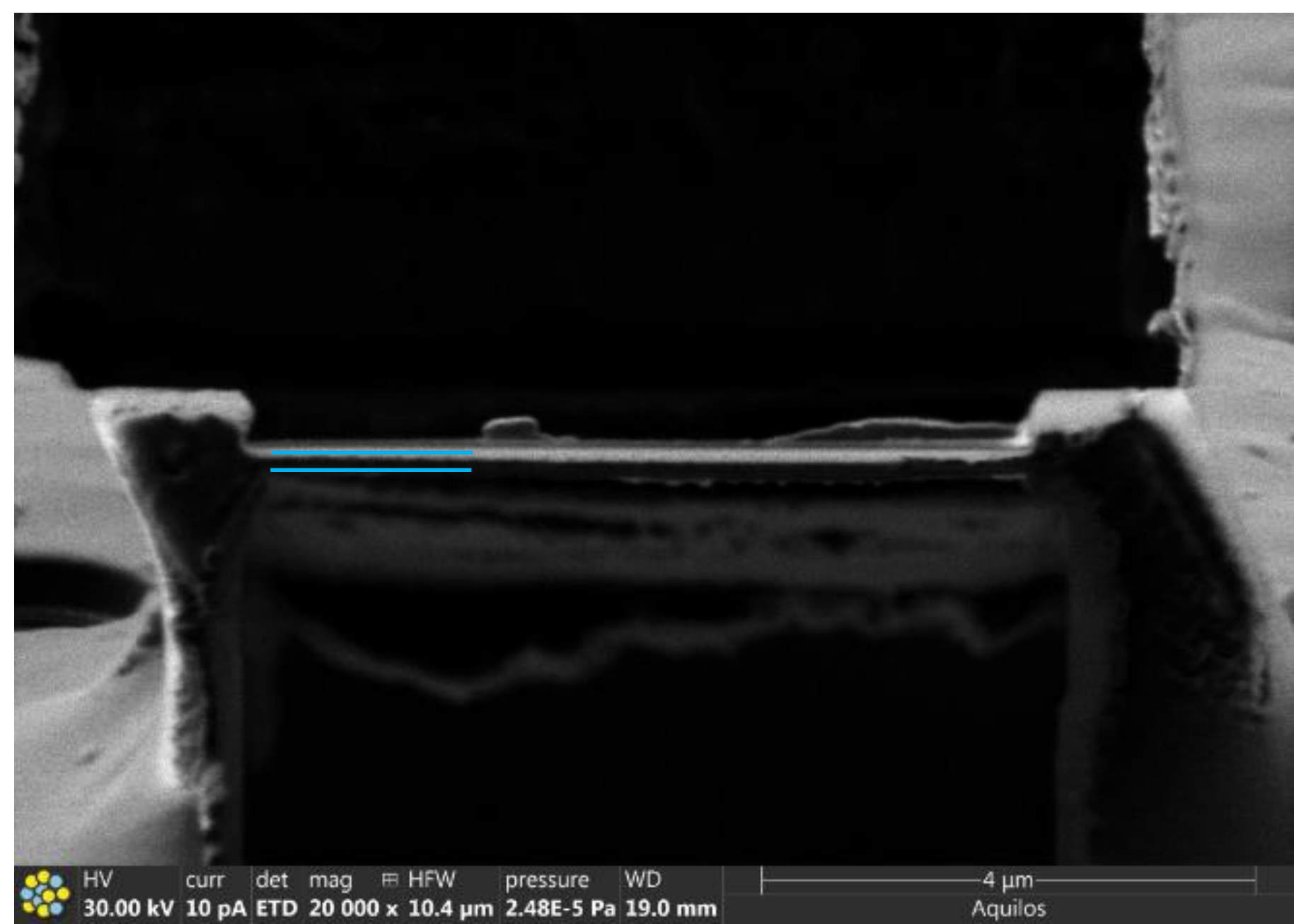

Single Frame

Maximum Projection

SI Figure 13 


\section{$260 \mathrm{~nm}$ lamella (0.95 x MFP) $200 \mathrm{kV}$}

Lamella

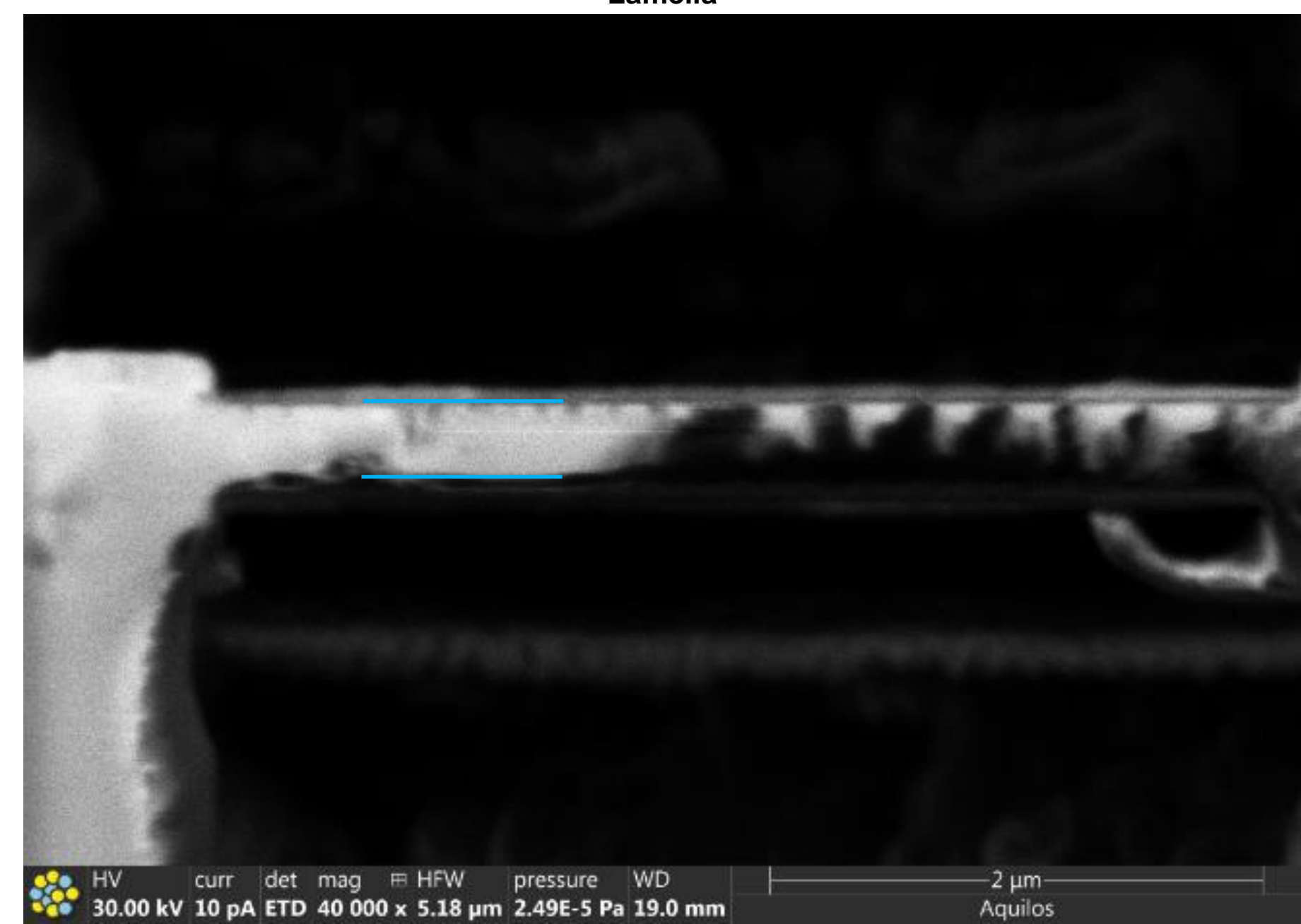

Single Frame

Maximum Projection

SI Figure 15 


\section{$260 \mathrm{~nm}$ lamella (0.95 x MFP) $200 \mathrm{kV}$}
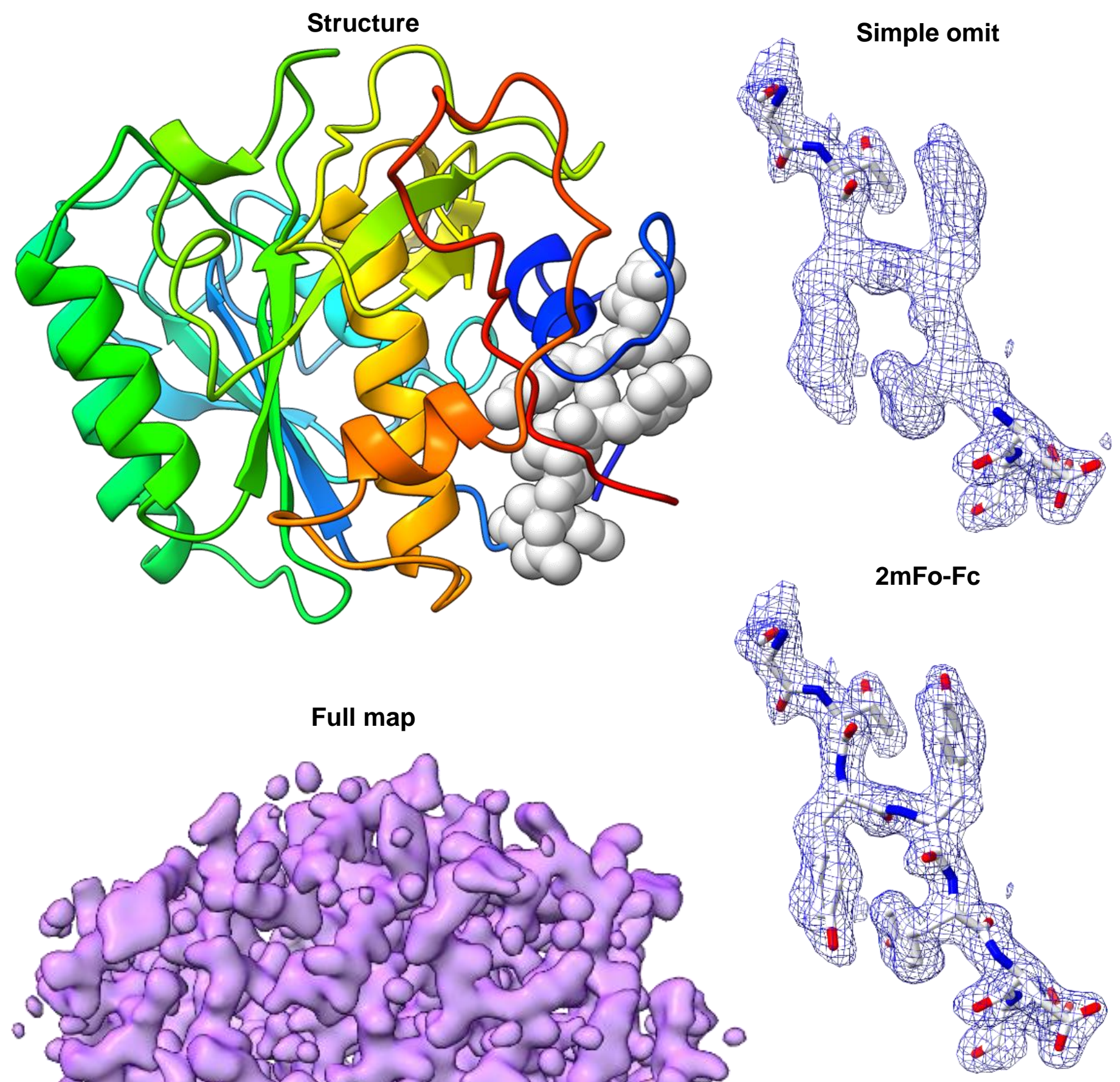

\section{SI Figure 16}

\section{Full map}

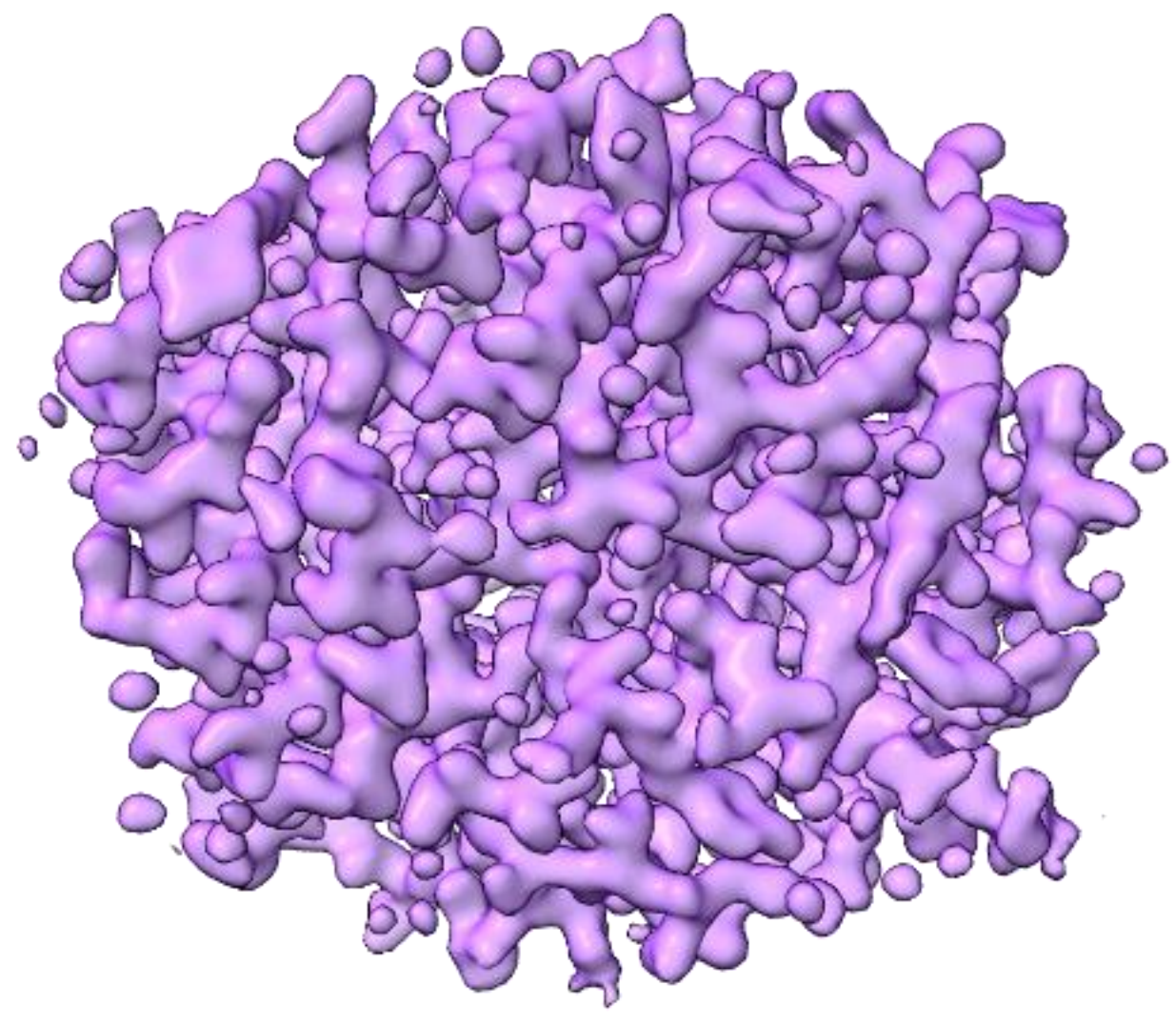


Lamella

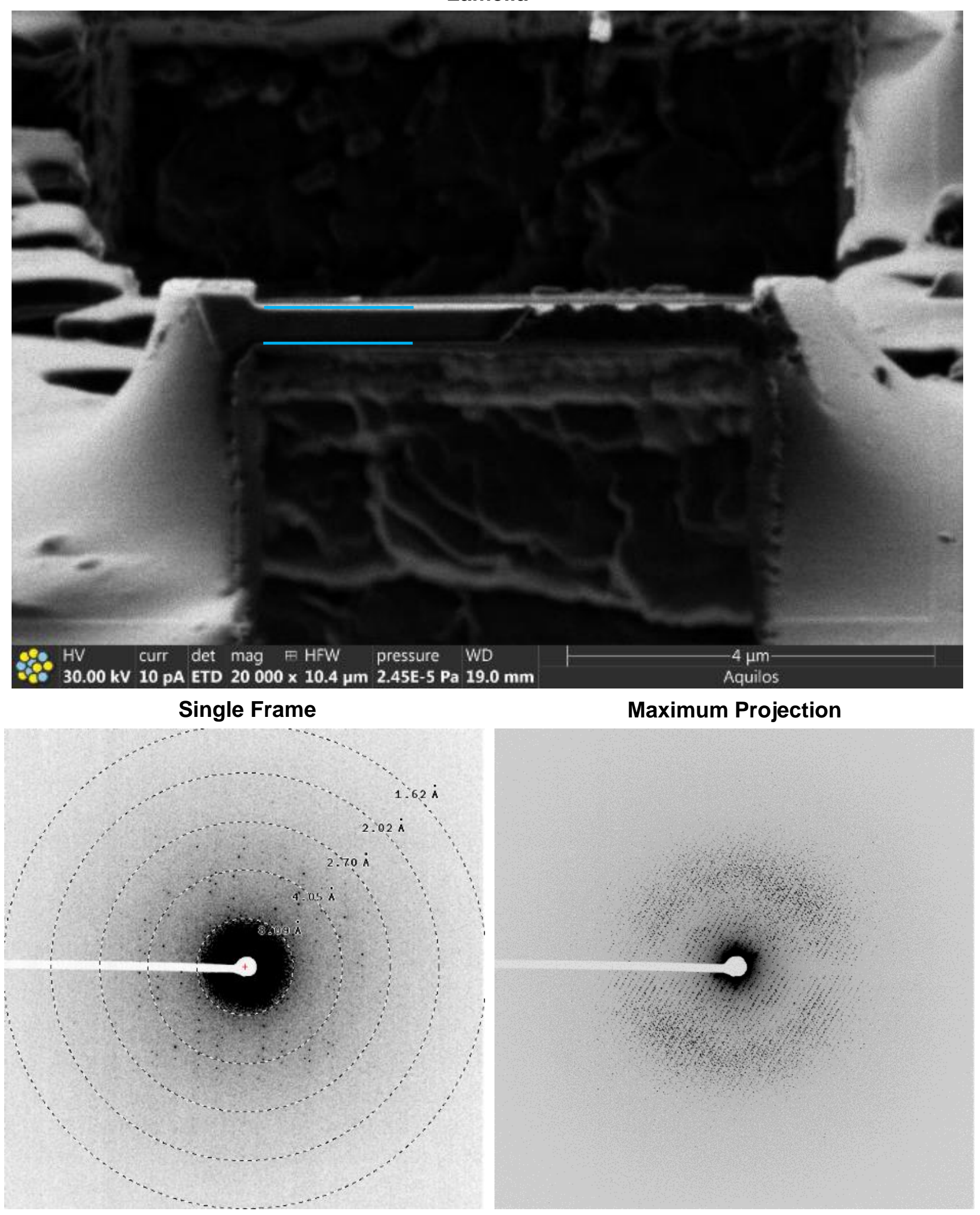

\section{SI Figure 17}


$460 \mathrm{~nm}$ lamella (1.7 x MFP) $200 \mathrm{kV}$
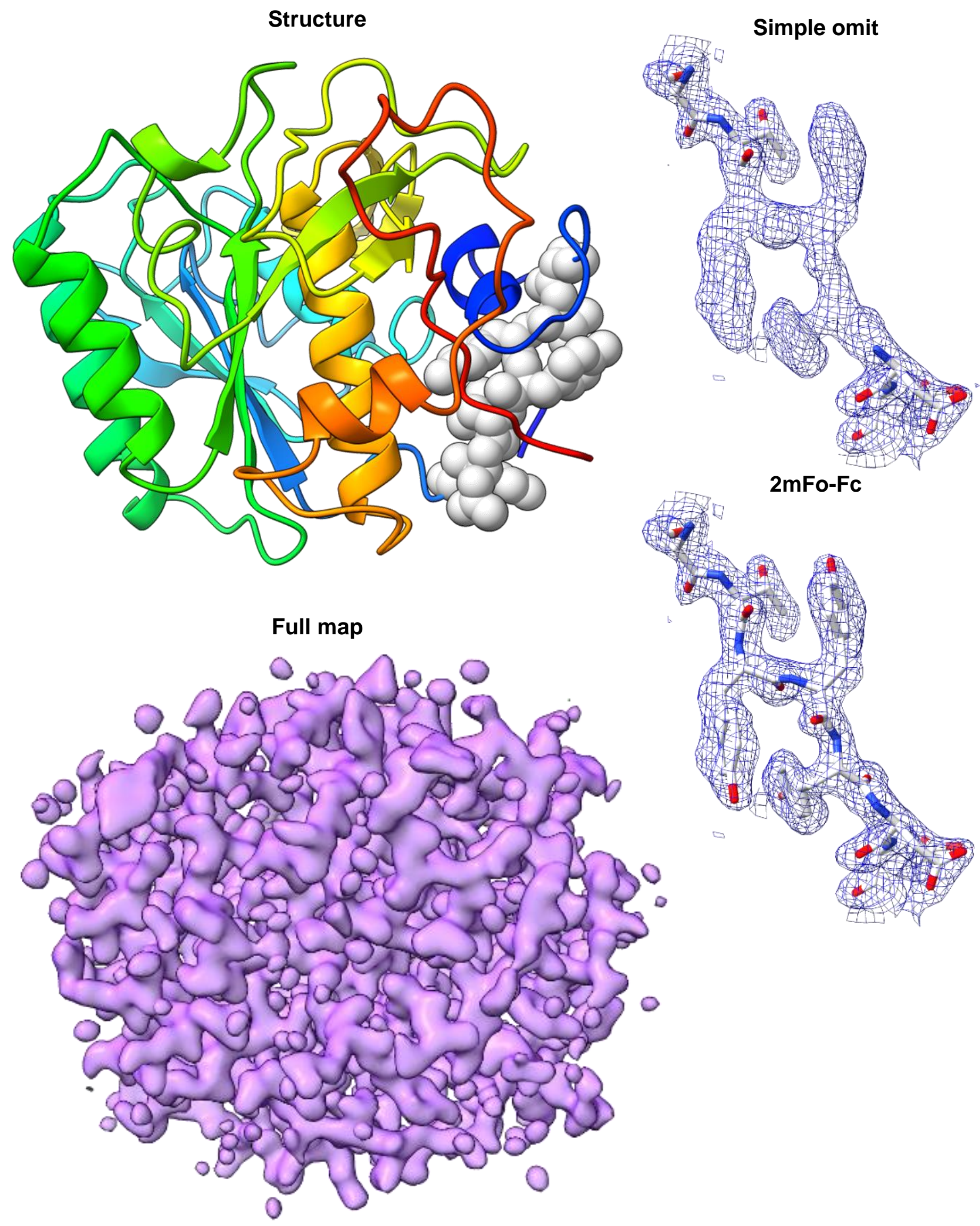

SI Figure 18 


\section{$530 \mathrm{~nm}$ lamella (1.9 x MFP) $200 \mathrm{kV}$}

\section{Lamella}

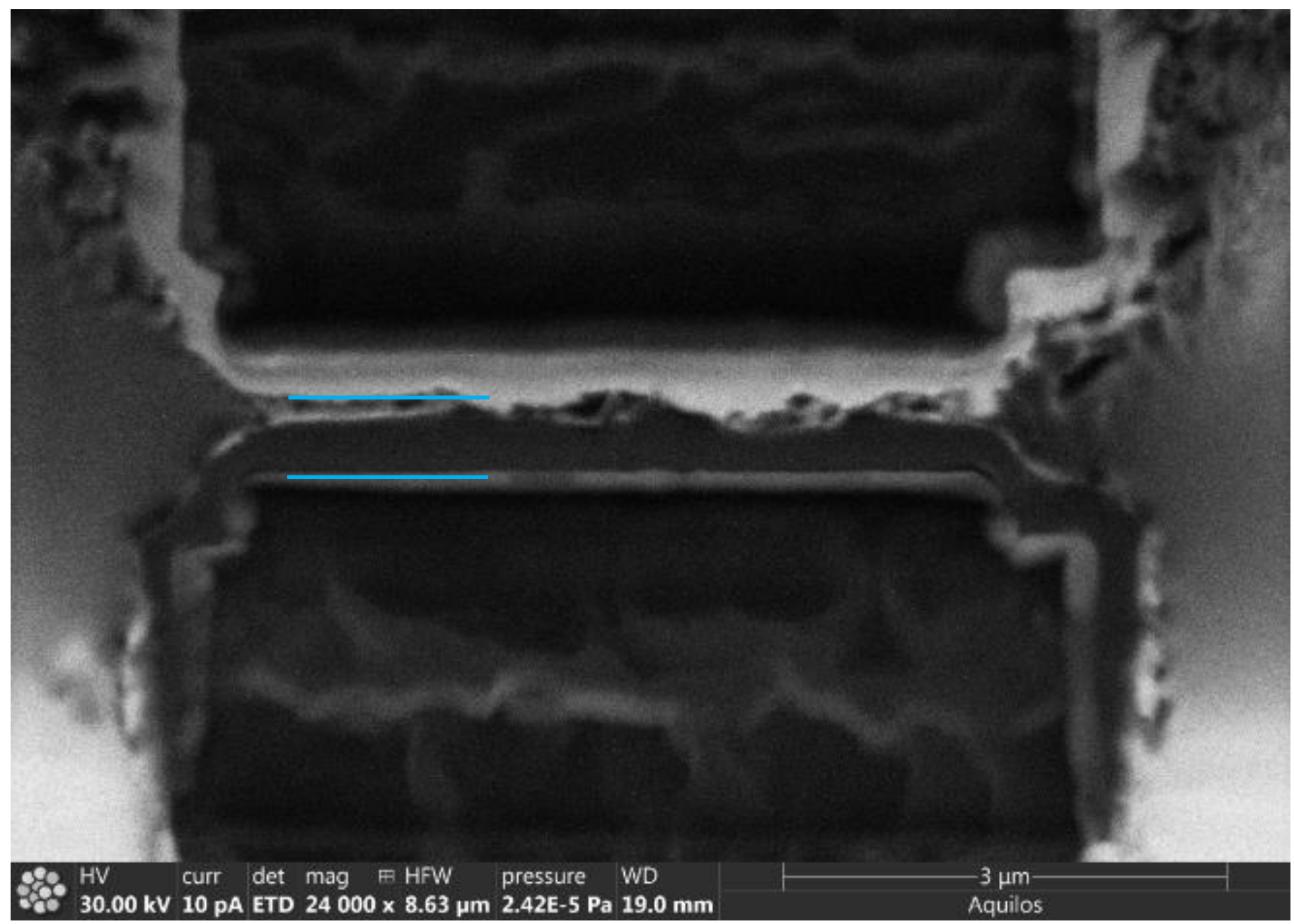

\section{Single Frame}

Maximum Projection

SI Figure 19 


\section{Structure}

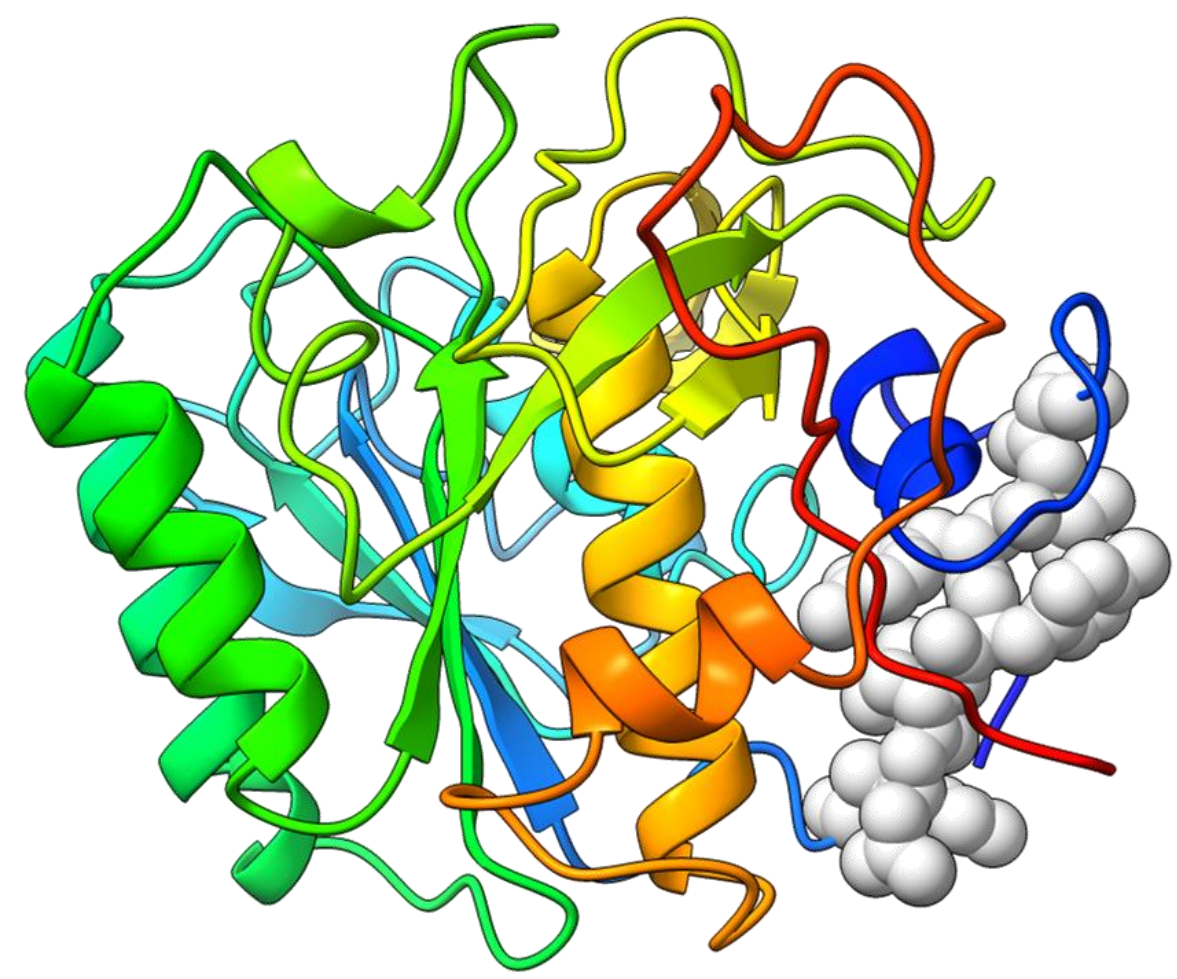

Simple omit

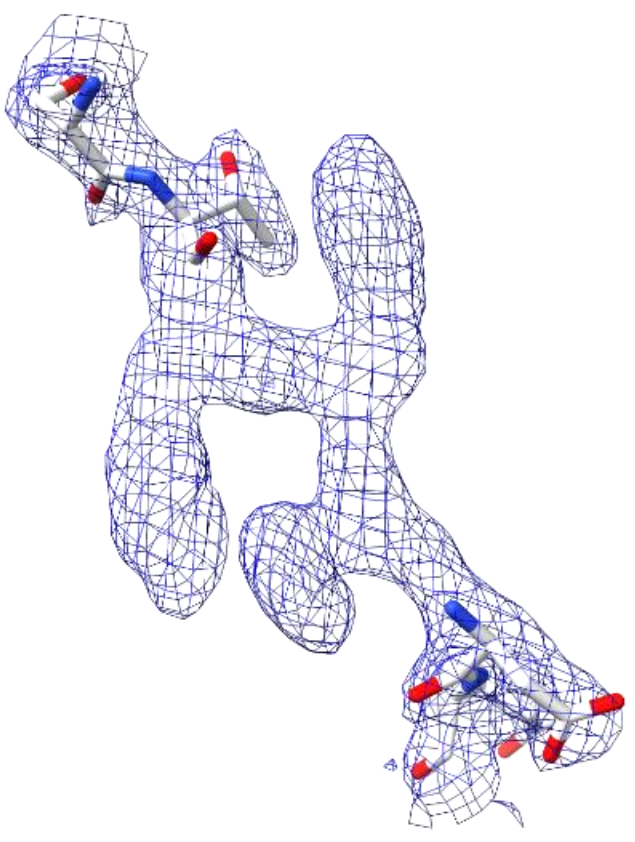

2mFo-Fc

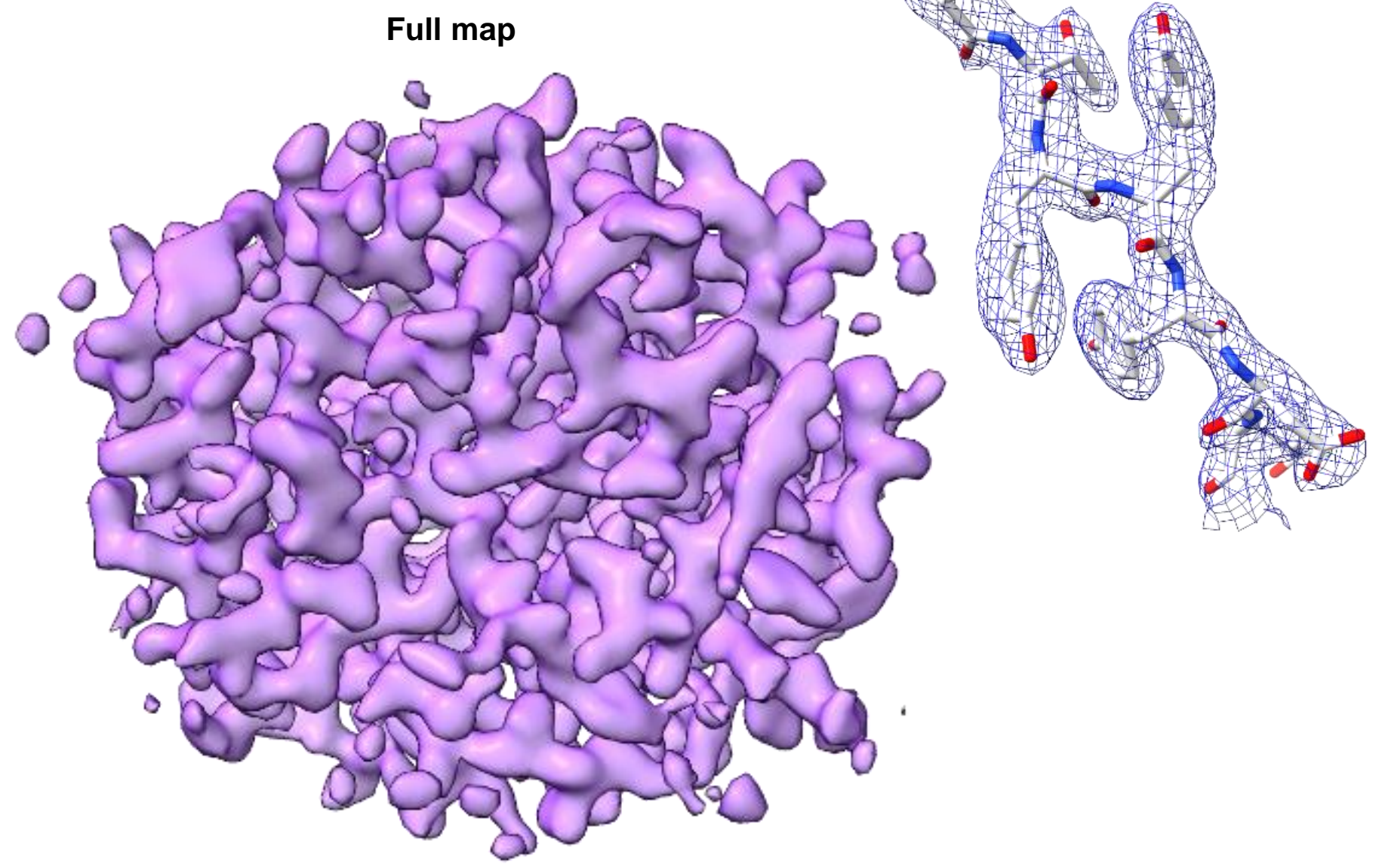

SI Figure 20 


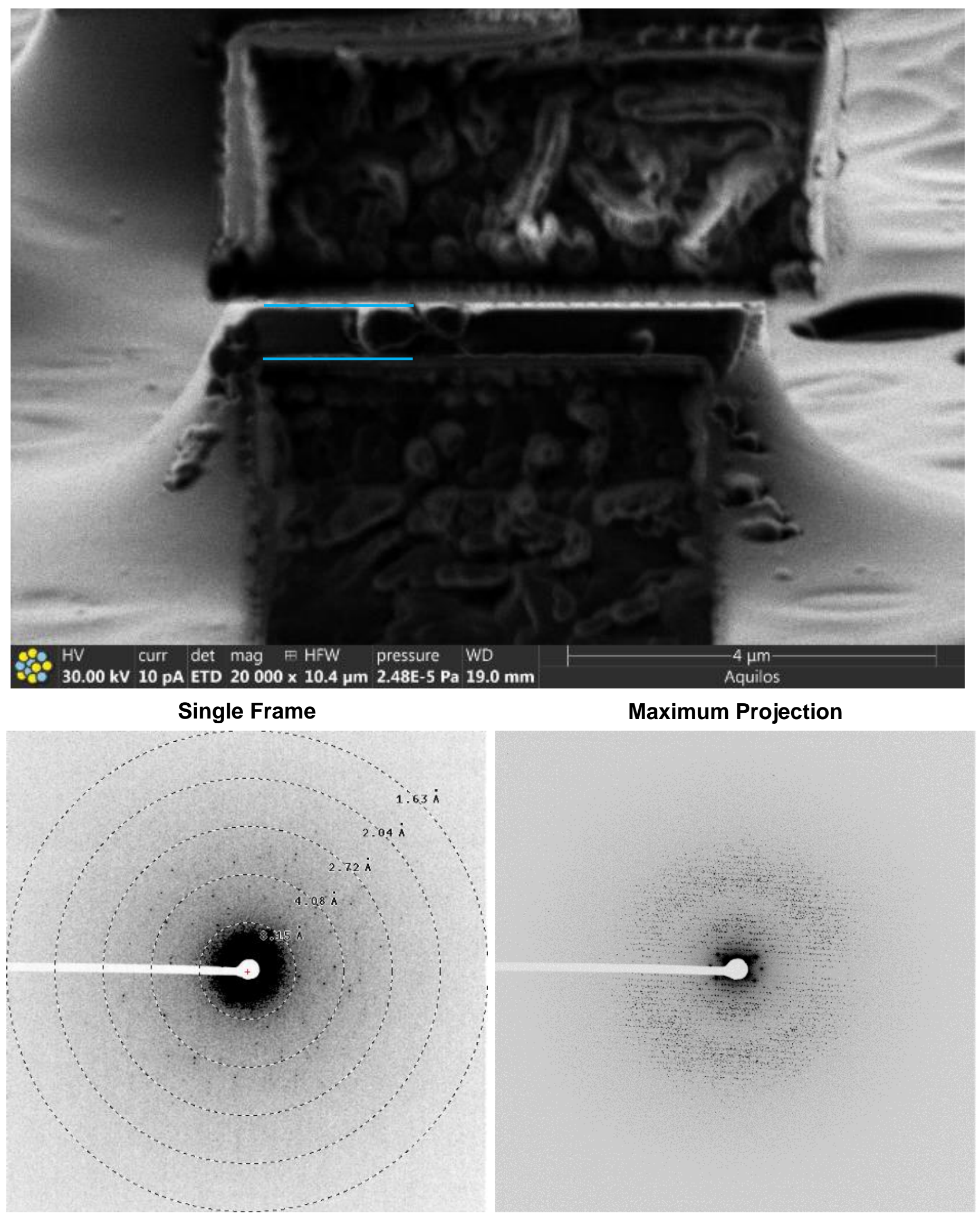

\section{SI Figure 21}




\section{$1400 \mathrm{~nm}$ lamella (5.14 x MFP) $200 \mathrm{kV}$}

Lamella

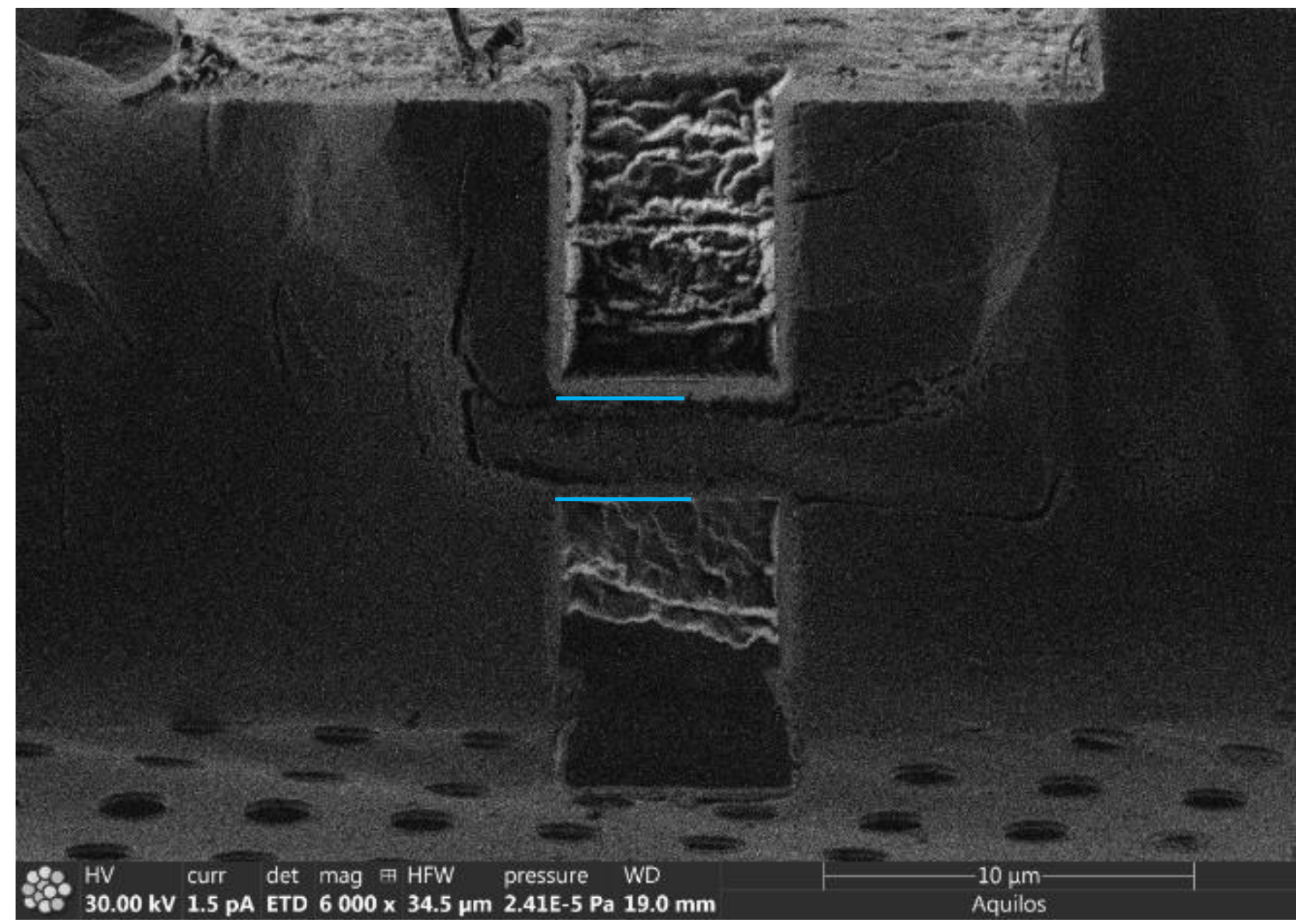

\section{Single Frame}

Maximum Projection

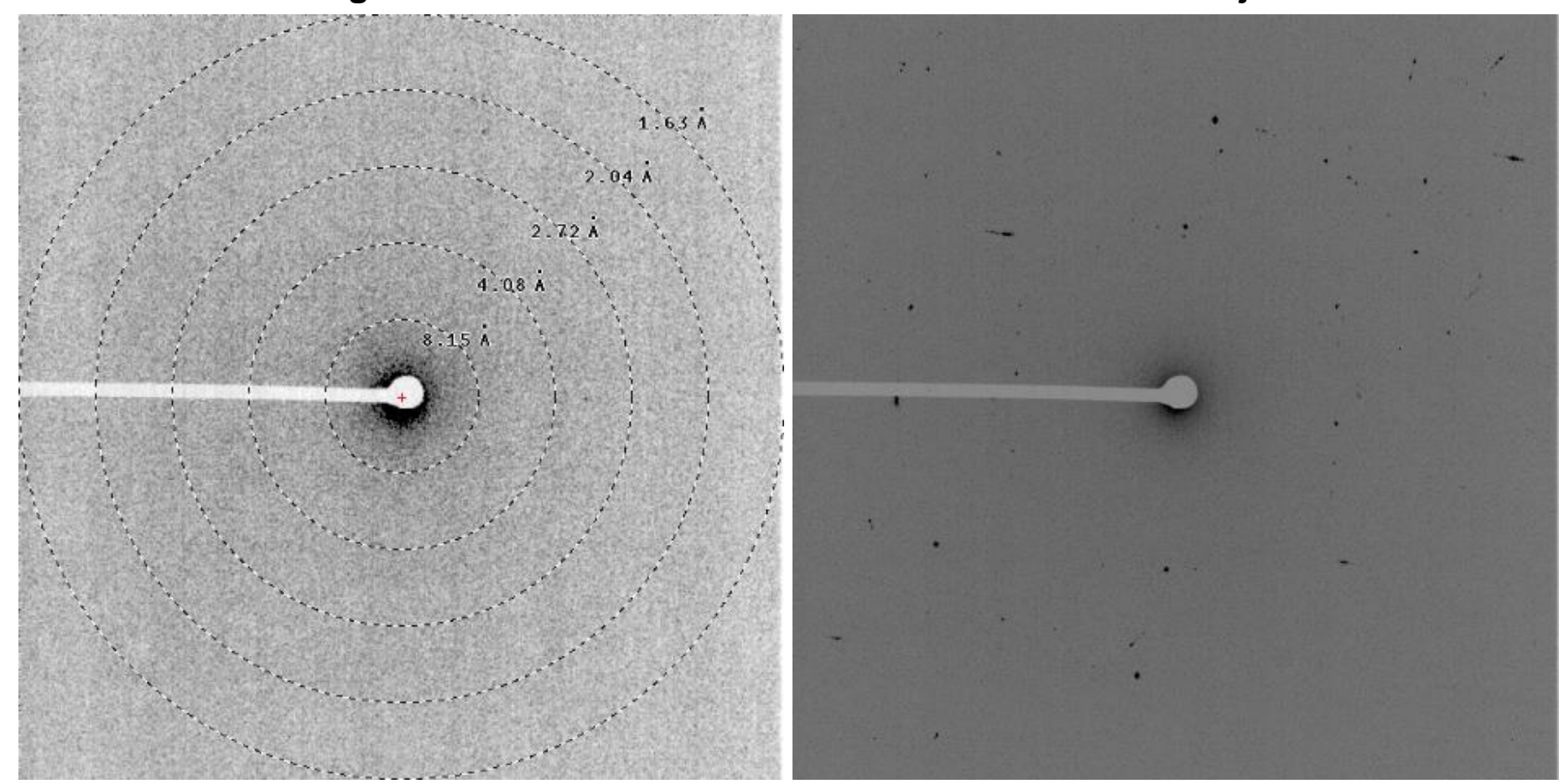

SI Figure 24 


\section{$150 \mathrm{~nm}$ lamella (0.47 x MFP) $300 \mathrm{kV}$}

Lamella

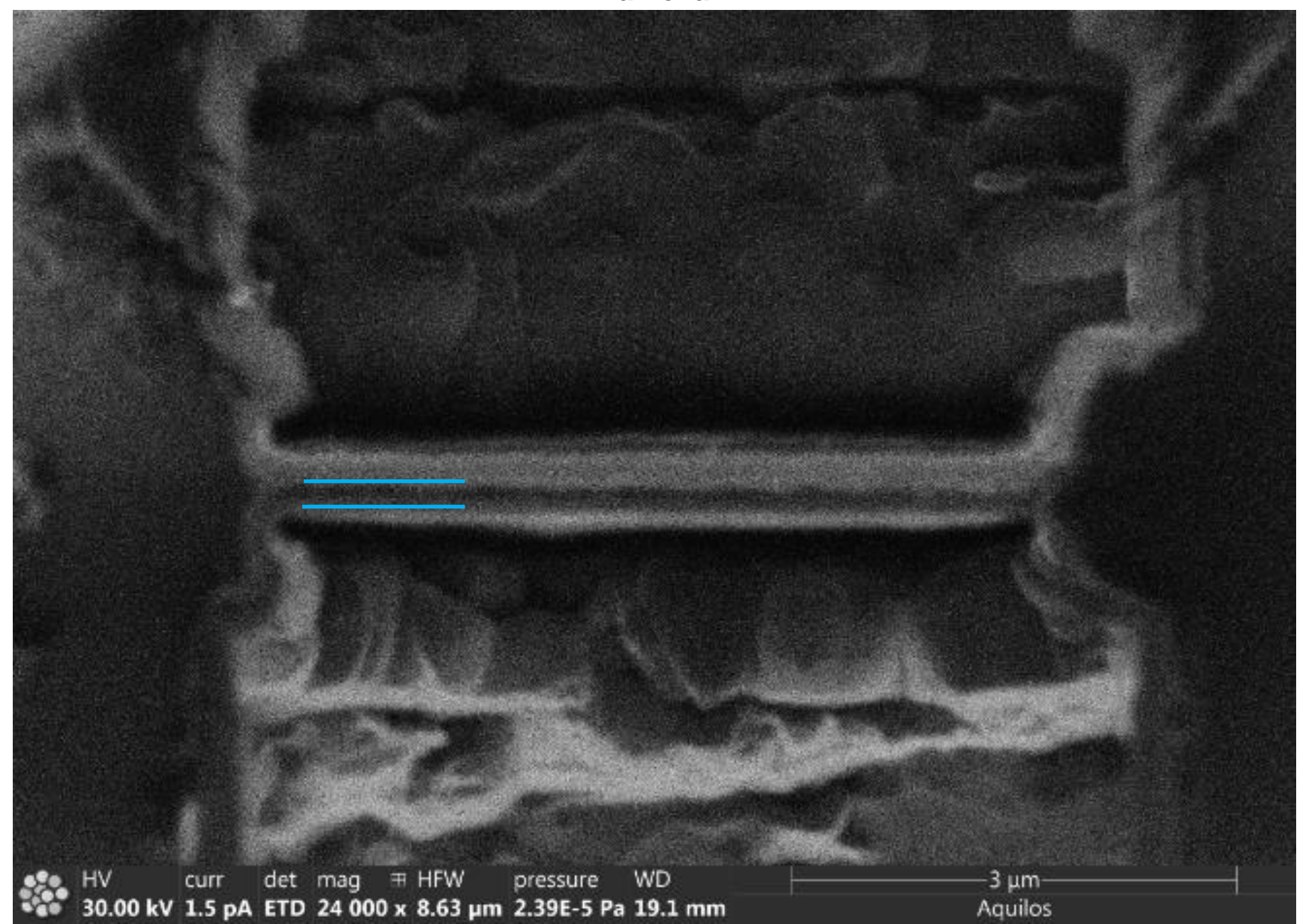

Single Frame

Maximum Projection

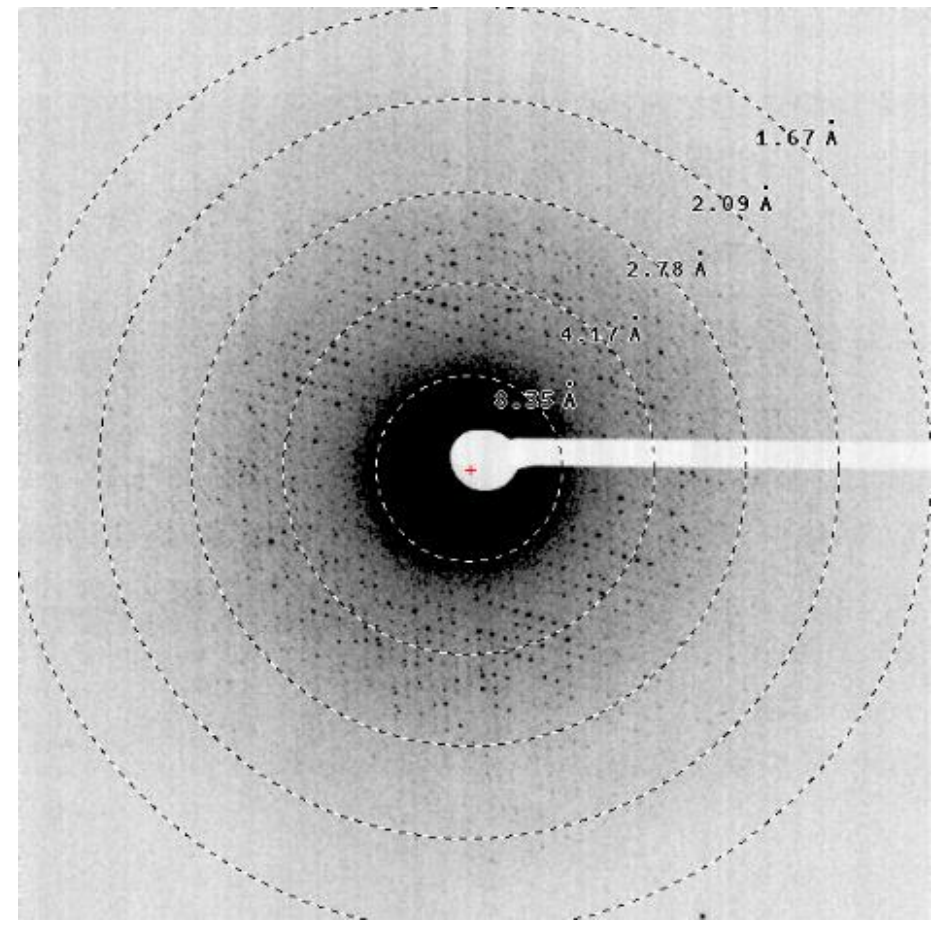

SI Figure 25 


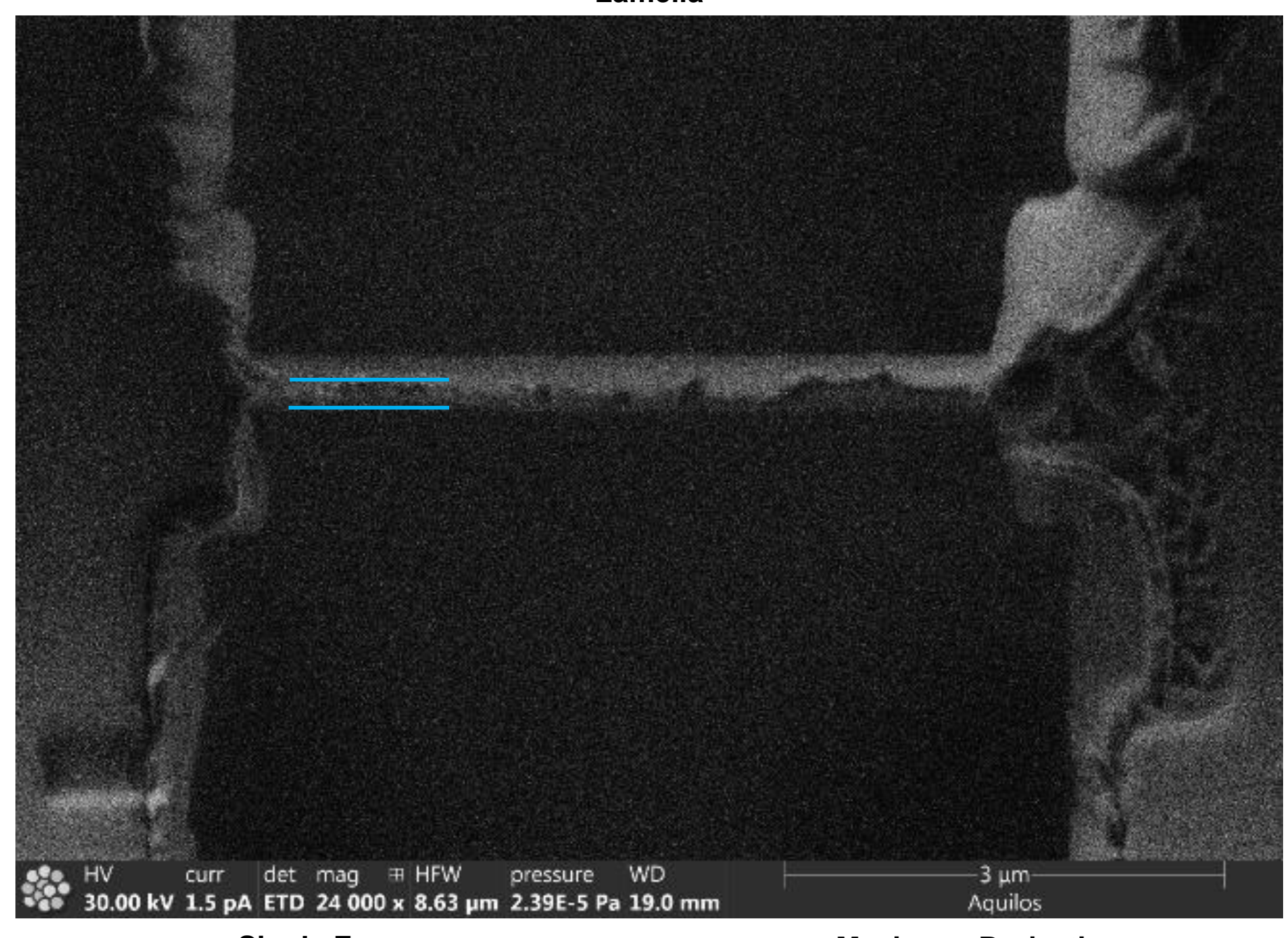

\section{Single Frame}

Maximum Projection

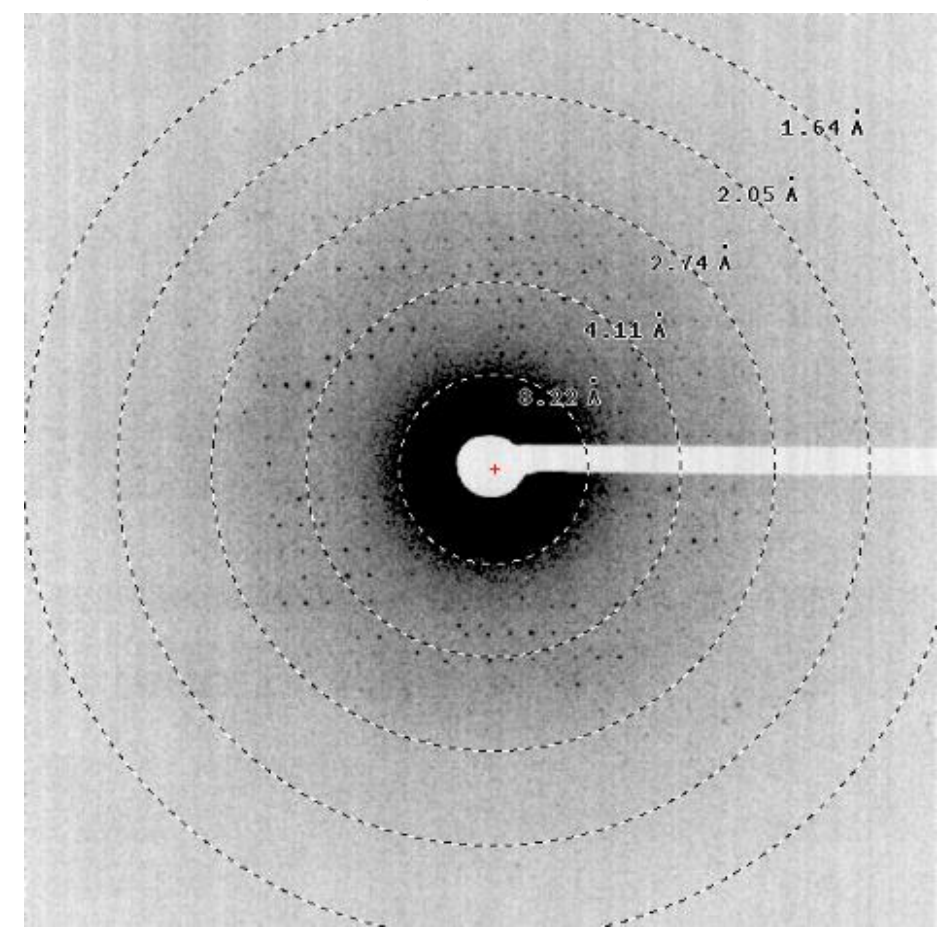

SI Figure 27 


\section{$320 \mathrm{~nm}$ lamella (1.0 x MFP) $300 \mathrm{kV}$}

\section{Lamella}

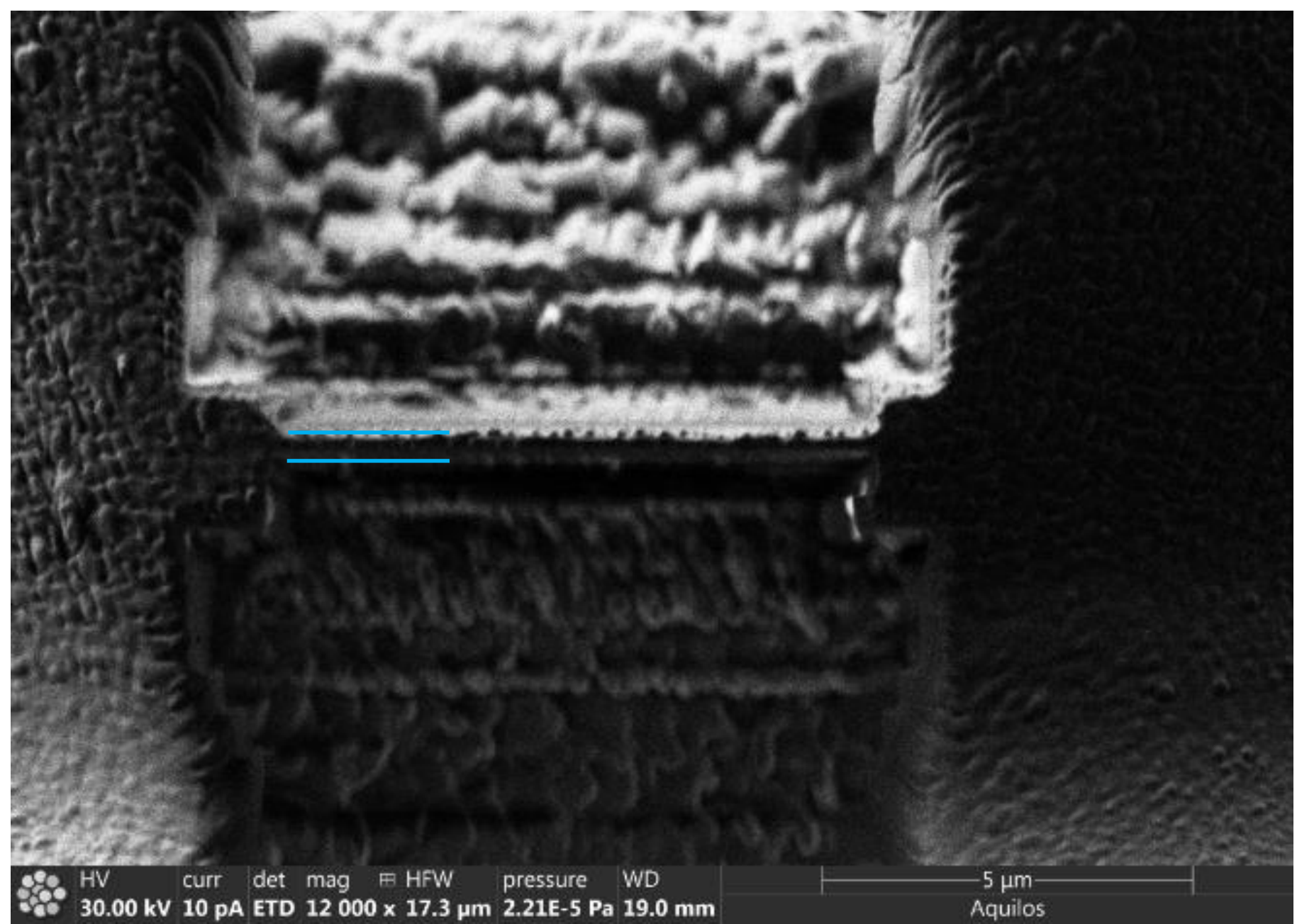

Single Frame

\section{Maximum Projection}




\section{$360 \mathrm{~nm}$ lamella (1.1 x MFP) $300 \mathrm{kV}$}

Lamella

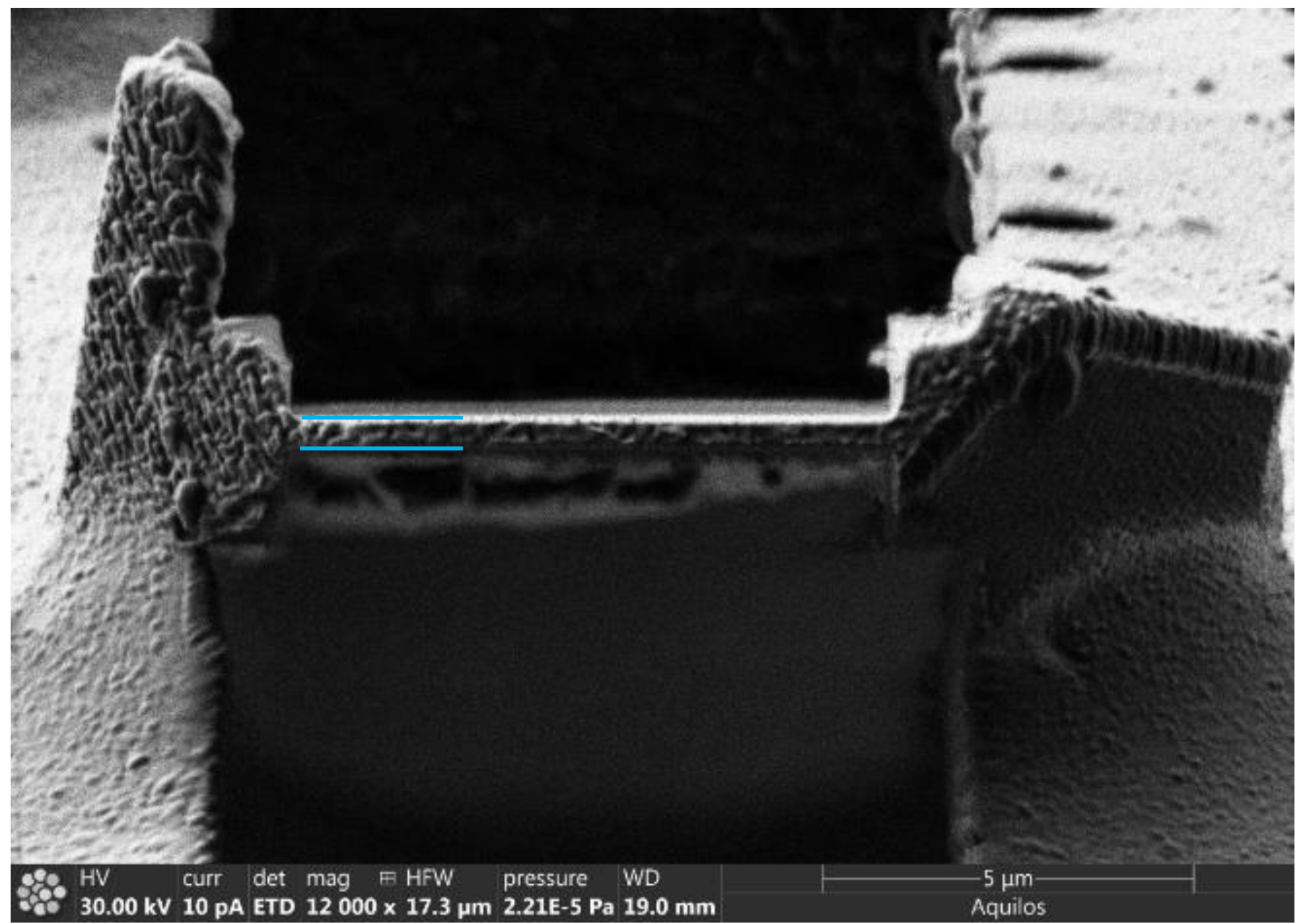

Single Frame

Maximum Projection

SI Figure 31

1:67

$208 \AA$

$2.78 \AA$

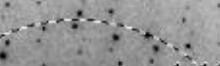
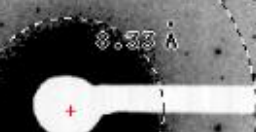


\section{$550 \mathrm{~nm}$ lamella (1.7 x MFP) $300 \mathrm{kV}$}

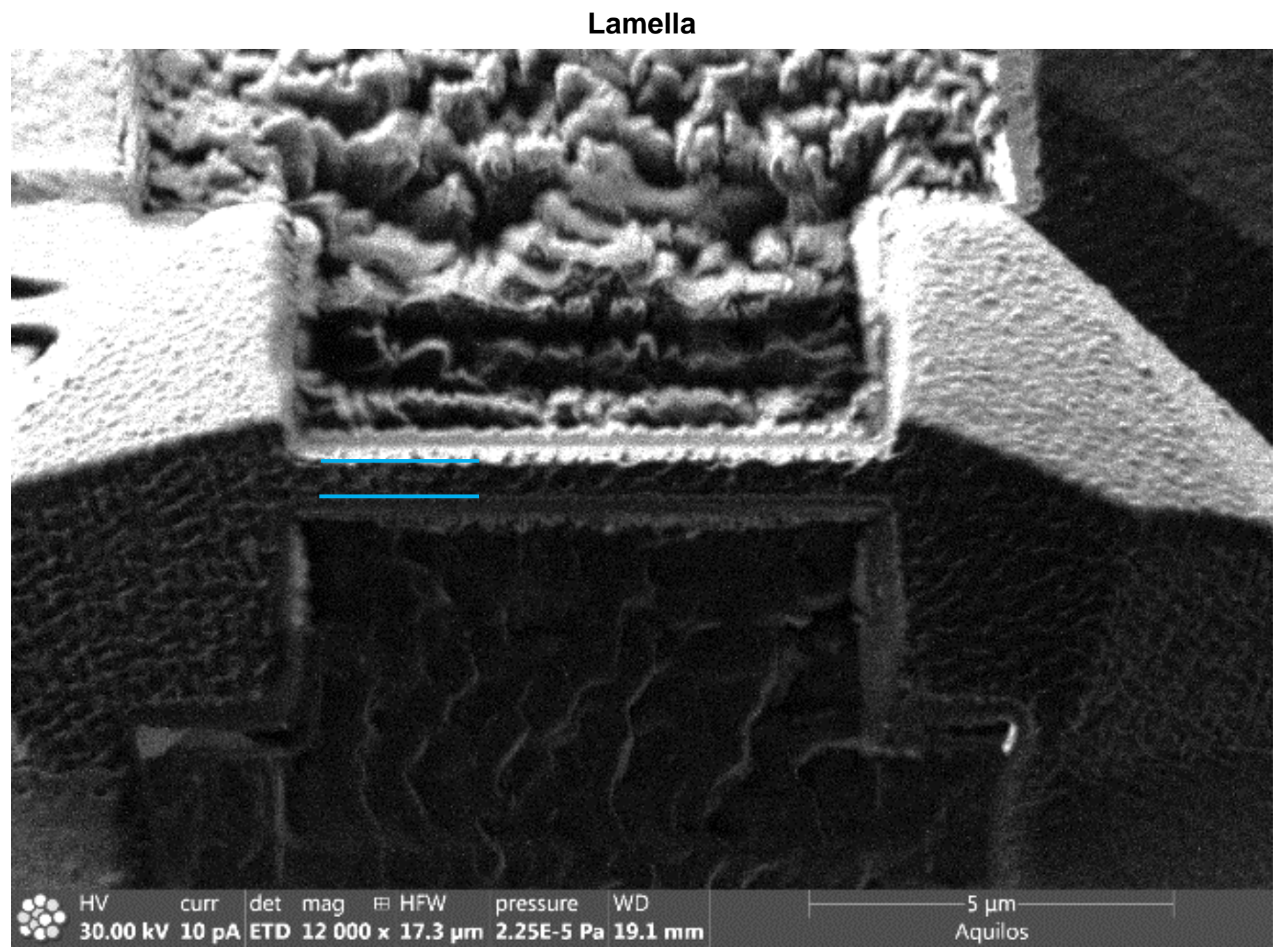

Single Frame

Maximum Projection

\section{SI Figure 33}

$1.68 \AA$

$2.10 \AA$

$2.80 \AA$

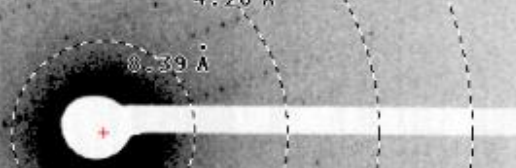



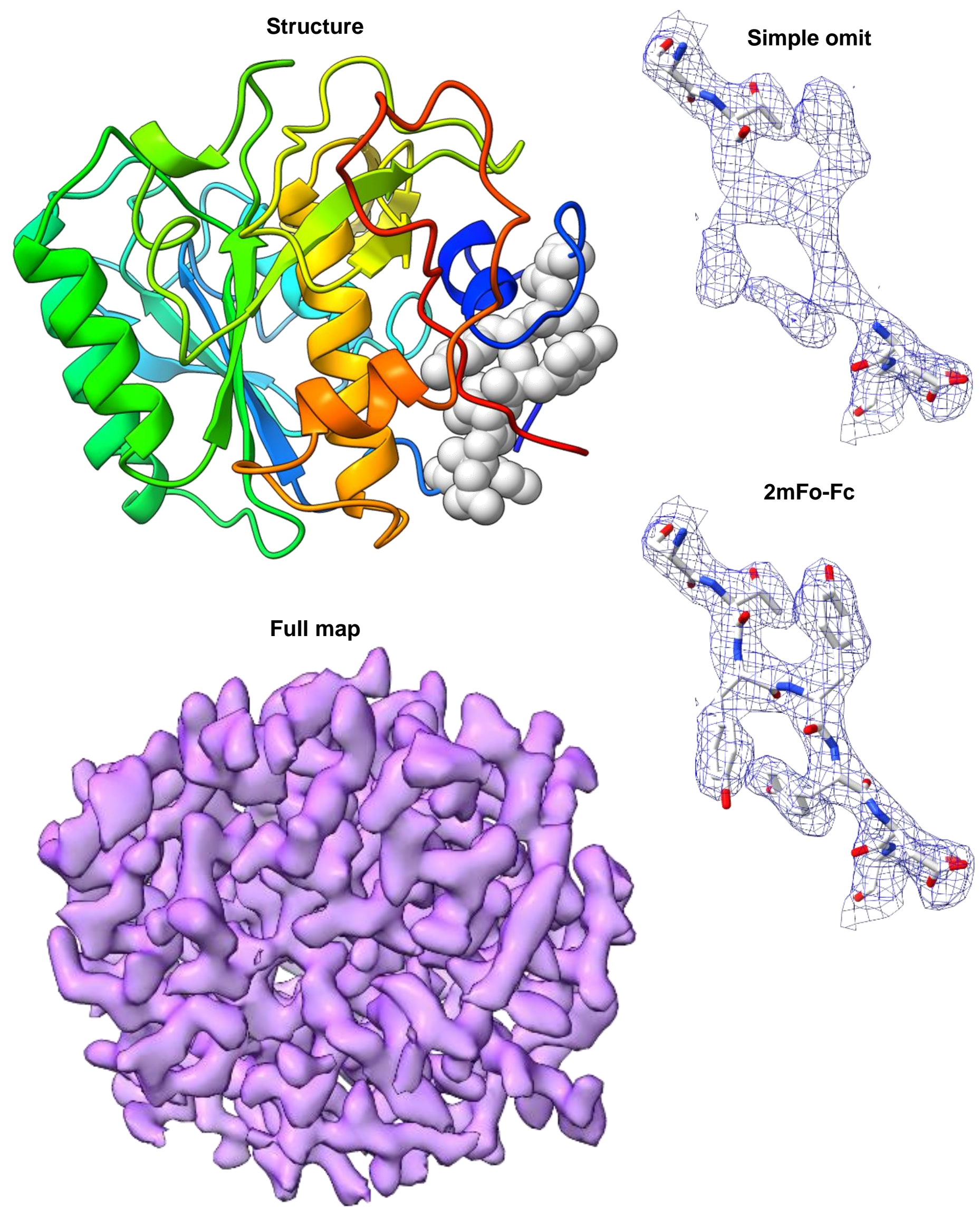

SI Figure 34 


\section{$880 \mathrm{~nm}$ lamella (2.8 x MFP) $300 \mathrm{kV}$}

Lamella

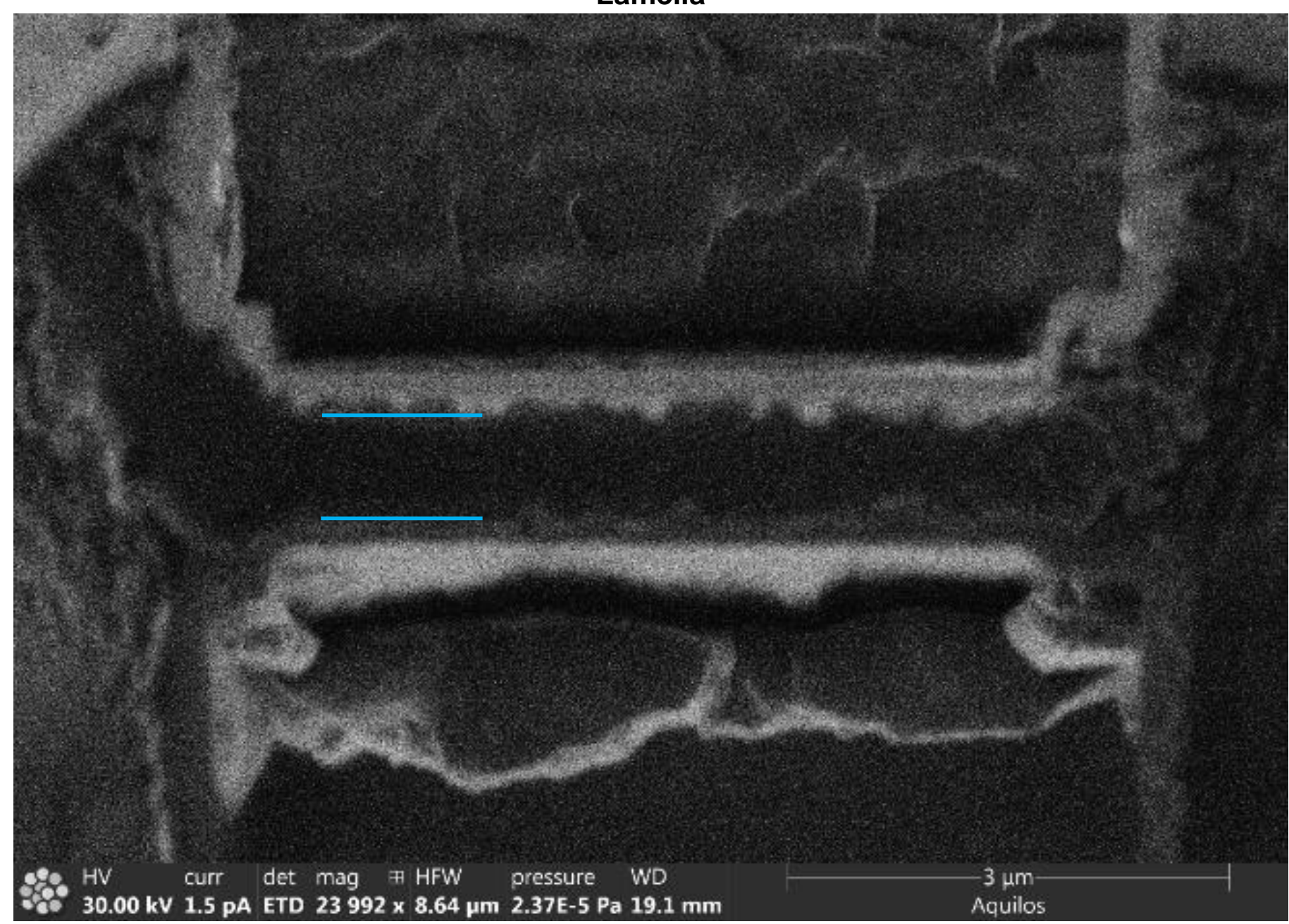

Single Frame

Maximum Projection

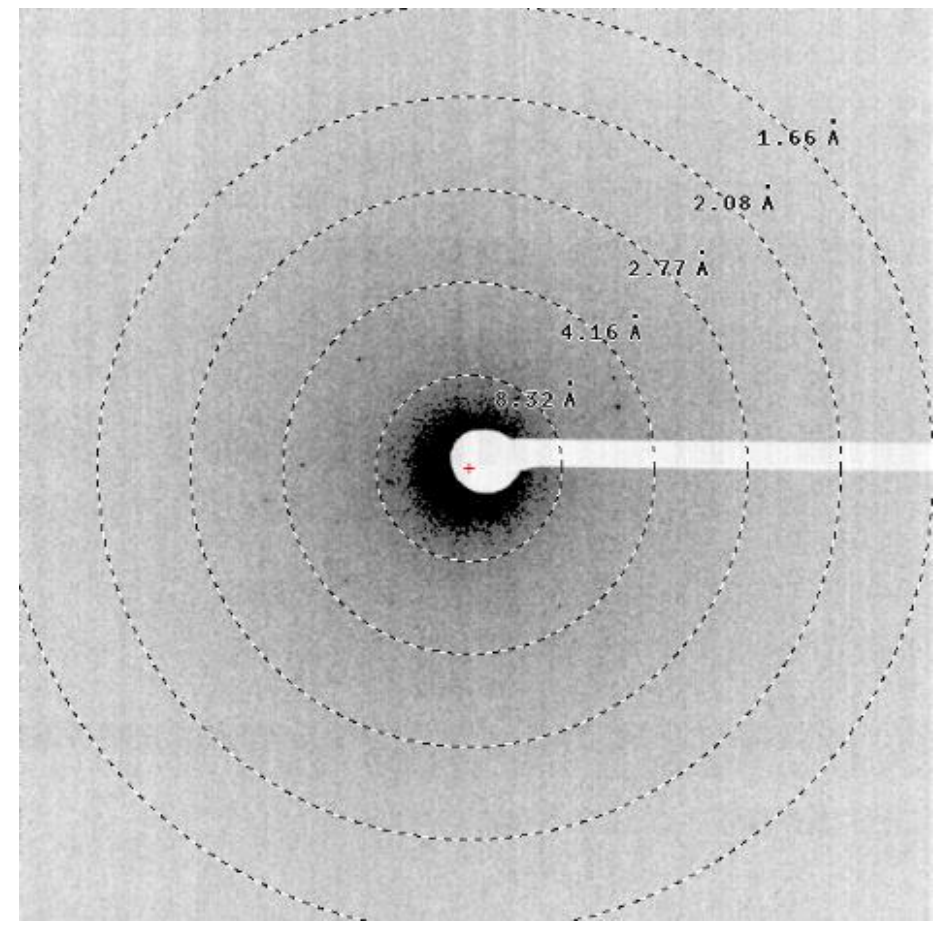

SI Figure 35 

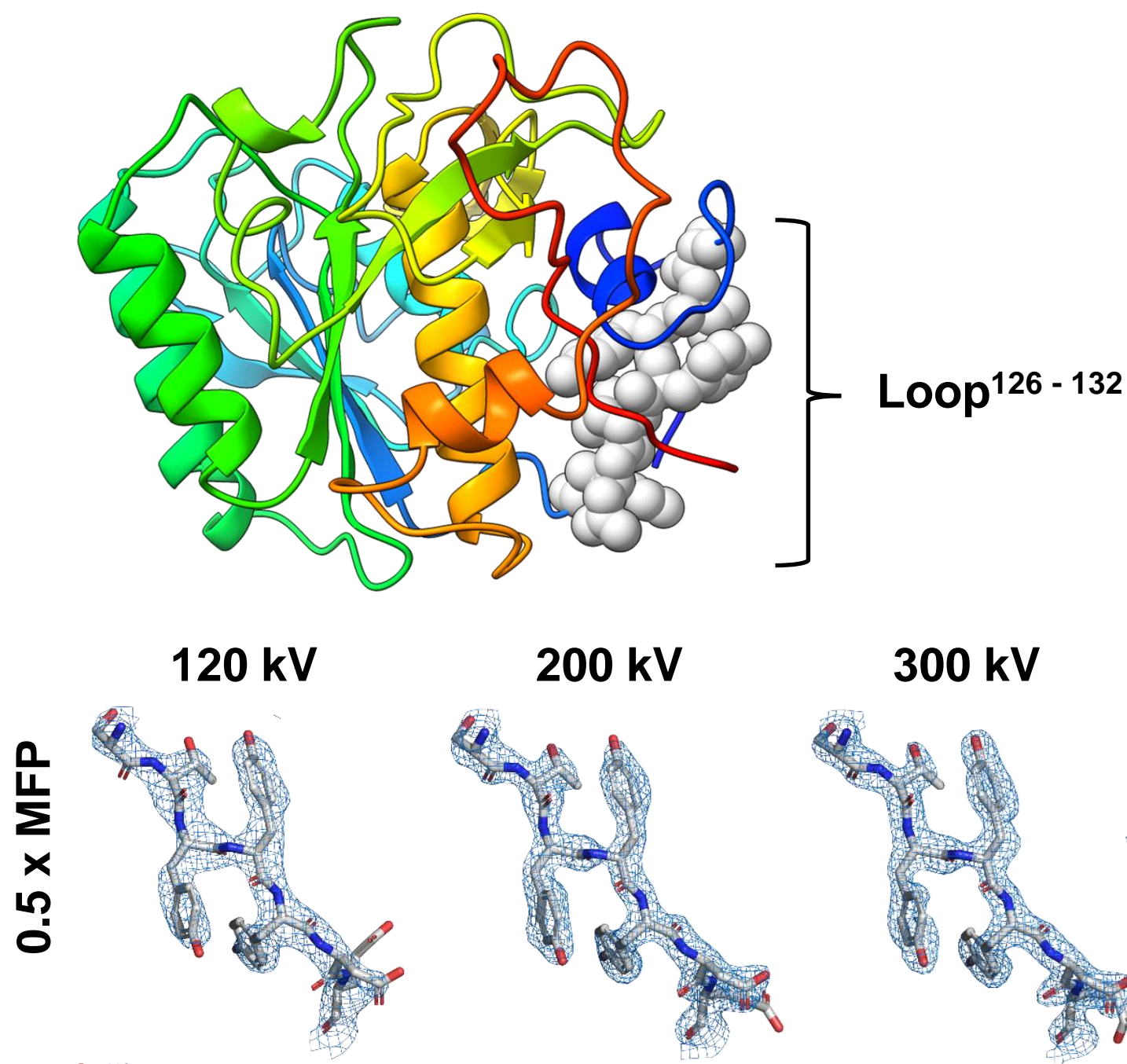

200 kV

$300 \mathrm{kV}$
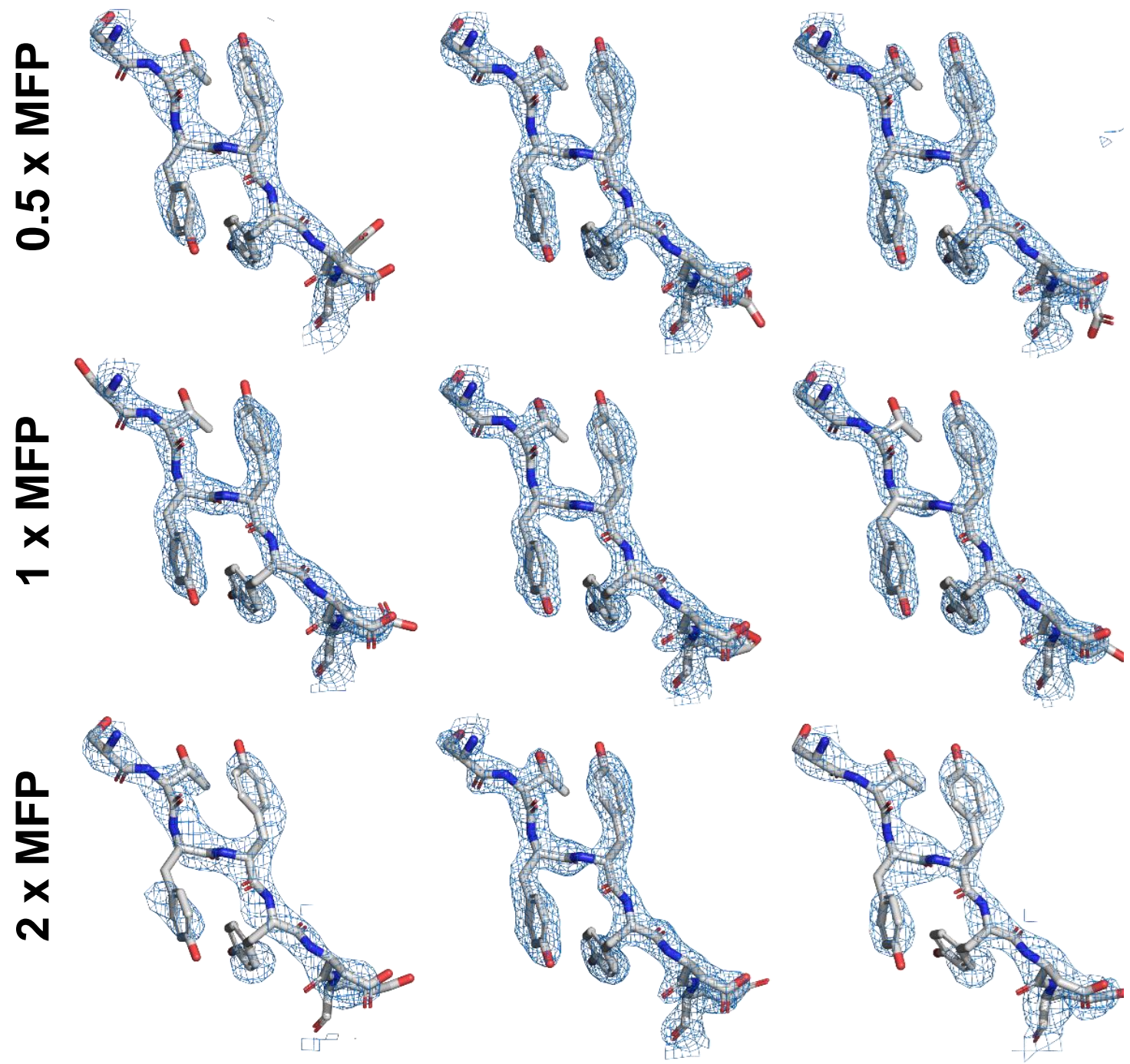

\section{SI Figure 37}




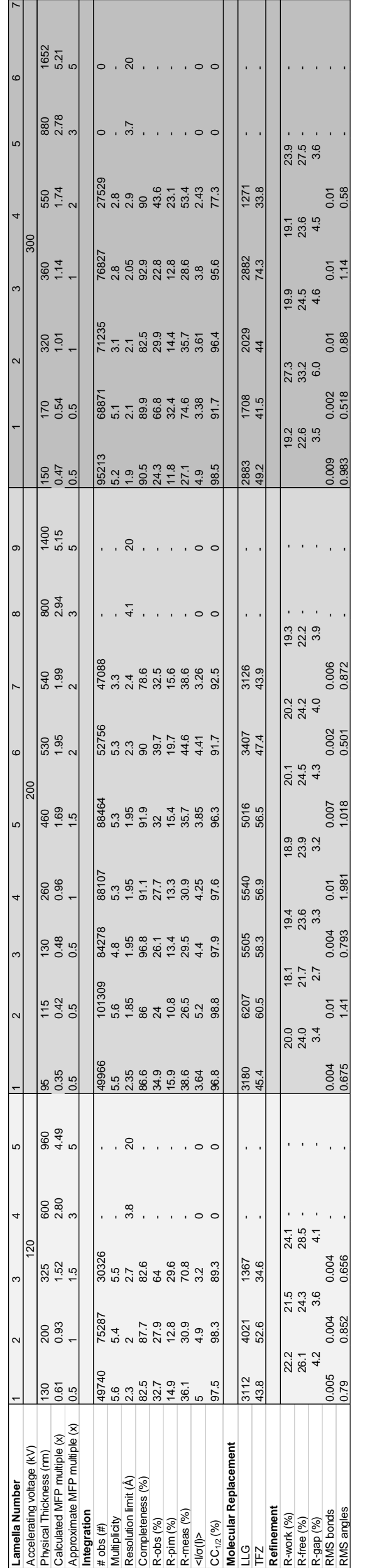

